\title{
Dieter Wiefelspütz
}

\section{Das Gewaltverbot und seine Durchbrechungen: Aktuelle Tendenzen im Völkerrecht}

\section{Die Bindung deutscher Staatsorgane an das Völkerrecht}

Beim Einsatz bewaffneter deutscher Streitkräfte im Ausland sind die Normen des Grundgesetzes, aber auch die Vorschriften des Völkervertragsrechts und die allgemeinen Regeln des Völkerrechts (Art. 25 GG) zu beachten und einzuhalten. ${ }^{1}$ Im Beschluss des Bundesverfassungsgerichts vom 26. Oktober 2004 (-2BvR 955/00 -, - 2 BvR 1038/01 -) heißt es dazu:

»Die deutschen Staatsorgane sind gemäß Art. 20 Abs. 3 GG an das Völkerrecht gebunden, das als Völkervertragsrecht nach Art. 59 Abs. 2 Satz 1 GG und mit seinen allgemeinen Regeln insbesondere als Völkergewohnheitsrecht nach Art. 25 Satz 1 GG innerstaatlich Geltung beansprucht. (...) Das Grundgesetz stellt die Staatsorgane mittelbar in den Dienst der Durchsetzung des Völkerrechts und vermindert dadurch das Risiko der Nichtbefolgung internationalen Rechts (vgl. Beschluss des Zweiten Senats des Bundesverfassungsgerichts vom 14. Oktober $2004-2$ BvR 1481/04 -, im Umdruck S. 30 und auch BVerfGE 109, $13<24>$; 109, $38<50>$ ). Eine solche verfassungsunmittelbare Pflicht ist nach deutschem Verfassungsrecht allerdings nicht unbesehen für jede beliebige Bestimmung des Völkerrechts anzunehmen, sondern nur, soweit es dem in den Art. 23 bis 26 GG sowie in den Art. 1 Abs. 2, Art. 16 Abs. 2 Satz 2 GG niedergelegten Konzept des Grundgesetzes entspricht. Das Grundgesetz will die Öffnung der innerstaatlichen Rechtsordnung für das Völkerrecht und die internationale Zusammenarbeit in den Formen einer kontrollierten Bindung; es ordnet nicht die Unterwerfung der deutschen Rechtsordnung unter die Völkerrechtsordnung und den unbedingten Geltungsvorrang von Völkerrecht vor dem Verfassungsrecht an, sondern will den Respekt vor friedens- und freiheitswahrenden internationalen Organisationen und dem Völkerrecht erhöhen, ohne die letzte Verantwortung für die Achtung der Würde des Menschen und die Beachtung der Grundrechte durch die deutsche öffentliche Gewalt aus der Hand zu geben (vgl. Beschluss des Zweiten Senats des Bundesverfassungsgerichts vom 14. Oktober 2004 - 2 BvR 1481/04 -, im Umdruck S. 17). $\ll^{2}$

1 Vgl. Stefan Oeter, »Einsatzarten der Streitkräfte außer zur Verteidigung « in: Neue Zeitschrift für Webrrecht 2000, S. 89 (94); Torsten Stein / Christian von Buttlar, Völkerrecht, 11. Aufl., Köln/Berlin/München 2005, Rdnr. 189 ff.

2 BVerfGE 112, S. 1 (24 ff). 


\section{Das Gewaltverbot des Art. 2 Ziff. 4 SVN}

Das umfassende Verbot der Anwendung und Androhung militärischer Gewalt ${ }^{3}$ gehört zu den großen Errungenschaften der Entwicklung des Völkerrechts in den vergangenen Jahrzehnten. ${ }^{4}$ Als die "grundlegende Regel des heutigen Völkerrechts « als eine »der größten Errungenschaften in der Völkerrechtsentwicklung seit dem 2. Weltkrieg ${ }^{6}$, als »basic principal of customary international law ${ }^{7}$, als Eckpfeiler des gegenwärtigen Völkergewohnheitsrechts ${ }^{8}$ und als einer der »leitenden Grundsätze «9 der VN wird das in Art. 2 Ziff. 4 SVN normierte absolute Gewaltverbot ${ }^{10}$ verstanden, das den Staaten die Gewaltanwendung in ihren internationalen Beziehungen verbietet. ${ }^{11}$ Das Gewaltverbot hat den Zweck, die territoriale Integrität der Staaten und ihre politische Handlungsfähigkeit zu sichern. ${ }^{12}$ Überwiegend wird das Ge-

3 Zur Geschichte des Gewaltverbots instruktiv: Bardo Fassbender, »Die Gegenwartskrise des völkerrechtlichen Gewaltverbotes vor dem Hintergrund der geschichtlichen Entwicklung in: Europäische Grundrechte-Zeitschrift 31 (2004), 9/12, S. $241 \mathrm{ff}$.

4 Matthias Herdegen, Völkerrecht, 4. Aufl., München 2005, \$34 Rdnr. 1.

5 Nach Rudolf Geiger, Grundgesetz und Völkerrecht, 3. Aufl., München 2002, \64 I 1 (S. 352), handelt es sich beim Gewaltverbot um »den harten Kern des völkerrechtlichen jus cogens«. Oliver Dörr, »Gewalt und Gewaltverbot im modernen Völkerrecht « in: Aus Politik und Zeitgeschichte: Beil. zur Wochenzeitung Das Parlament 54 (2004), 43 vom 18.10.2004, S. 14 (20), spricht von der "zentralen Norm der internationalen Ordnung «.

6 Stephan Hobe, »NATO-Intervention im Kosovo: Rückkehr zur Lehre vom gerechten Krieg? « in: Bernhard Kempen, (Hg.), Die Macht des Geistes: Festschrift für Hartmut Schiedermair, Heidelberg 2001, S. 819 (821).

7 Jost Delbrück, »The Fight Against Global Terrorism: Self-Defense or Collective Security as International Police Action? Some Comments on the International Legal Implications of the 'War Against Terrorism' « in: German Yearbook Of International Law 44 (2001), S. 9 (13).

8 Yoram Dinstein, »Comments on the Presentations by Nico Kirsch and Carsten Stahn« in: Christian Walter / Silja Vöneky / Volker Röben / Frank Schorkopf, (Hg.), Terrorism as a Challenge for National and International Law: Security versus Liberty?, Berlin u. a. 2004, S. 915; Hartmut Hillgenberg, »Gewaltverbot: Was gilt noch?« in: Jochen Abr. Frowein / Klaus Scharioth / Ingo Winkelmann / Rüdiger Wolfrum, (Hg.), Verhandeln für den Frieden - Negotiating for Peace, Liber Amicorum Tono Eitel, Berlin u. a. 2003, S. 141 (143): »Kernstück der Charta«.

9 Pierre-Marie Dupuy / Christian Tomuschat, »Warten auf den Schlag gegen Bagdad « in: Frankfurter Allgemeine Zeitung vom 31. Juli 2002.

10 Vgl. Albrecht Randelzhofer, in: Bruno Simma, (Hg.), The Charter of the United Nations, Volume I, Second Edition, Oxford u. a. 2002, Art. 2 Ziff. 4 Rdnr. 14; Horst Fischer, in: Knut Ipsen, Völkerrecht, 5. Aufl., München 2004, \59 Rdnr. 9 ff.; Dietrich Schindler, in: Dietrich Schindler / Kay Hailbronner, Die Grenzen des völkerrechtlichen Gewaltverbots, Berichte der Deutschen Gesellschaft für Völkerrecht, Heft 26, Heidelberg 1986, S. 11 ff.; Michael Bothe, in: Wolfgang Graf Vitzthum, (Hg.), Völkerrecht, 3. Aufl., Berlin 2004, 8. Abschnitt, Rdnr. 9 ff. m. w. N.

11 Rüdiger Wolfrum, »Irak - eine Krise auch für das System der kollektiven Sicherheit « in: http://www.mpil.de/de/Wolfrum/irak.pdf, S. 1.

12 Georg Dahm / Jost Delbrück / Rüdiger Wolfrum, Völkerrecht, Bd. I/3, Die Formen des völkerrechtlichen Handelns. Die inhaltliche Ordnung der internationalen Gemeinschaft, Berlin u. a. 2002, \$169 XI (S. 832). 
waltverbot in der Form, die es in Art. 2 Ziff. 4 SVN gefunden hat, zum universellen Völkergewohnheitsrecht gerechnet. ${ }^{13}$ Obwohl das Gewaltverbot des Art. 2 Ziff. 4 SVN mehrfach »totgesagt « wurde, ${ }^{14}$ ist es weiterhin die unverzichtbare zentrale Norm für die Gestaltung der internationalen Beziehungen.

Das völkerrechtliche Gewaltverbot geht weiter als das Verbot des Angriffskrieges. Unter Gewalt i. S. des Art. 2 Ziff. 4 SVN ist Waffengewalt (militärische Gewalt) zu verstehen. ${ }^{15}$ Das Verbot, Gewalt auf fremdem Staatsgebiet anzuwenden, gilt ohne Einschränkung hinsichtlich der Intensität der Gewaltanwendung. ${ }^{16}$ Keinem Staat zurechenbare »private « Gewalt ist vom Gewaltverbot nicht erfasst. ${ }^{17}$

Das Gewaltverbot bezieht sich nicht nur auf militärische Operationen gegen einen Staat, sondern erfasst sämtliche bewaffnete Handlungen, auch Aktionen gegen nichtstaatliche Akteure, weil auch in diesem Fall regelmäßig die territoriale Unversehrtheit des Aufenthaltsstaates verletzt wird..$^{18}$

Unstreitig ist, dass von Art. 2 Ziff. 4 SVN die Gewaltanwendung im Innern eines Staates nicht berührt wird. Insbesondere umfasst das Gewaltverbot des Art. 2 Ziff. 4 SVN in der Regel nicht die Durchsetzung der staatlichen Gebietshoheit. ${ }^{19}$ Jeder Staat hat das Recht, Grenzverletzungen notfalls gewaltsam zu verhindern. ${ }^{20}$ Das

13 ICJ, Case Concerning Military and Paramilitary Activities in and Against Nicaragua, Merits, ICJ Reports 1986, 14, para. 188; vgl. auch Oliver Dörr, »Das völkerrechtliche Gewaltverbot am Beginn des 21. Jahrhunderts - Was bleibt von Art. 2(4) UN-Charta?« in: Oliver Dörr, (Hg.), Ein Rechtslehrer in Berlin. Symposium für Albrecht Randelzhofer, Berlin u. a. 2004, S. 33 (37); Christian Schaller, Das Friedenssicherungsrecht im Kampf gegen den Terrorismus, Berlin 2004, S. 7; Rüdiger Wolfrum, »American-European Dialogue: Different Perceptions of International Law - Introduction « in: Zeitschrift für ausländisches öffentliches Recht und Völkerrecht 64 (2004), S. 255 (257); Dieter Dörr / Dorit Bosch, »Der praktische Fall - Völkerrecht: Aufmarsch vor dem Irak « in: Juristische Schulung 2003, S. 477 (482); Hans-Georg Dederer, »Krieg gegen Terror « in: Juristenzeitung 2004, S. 421; Sebastian Weinzierl, »Vorlesungs-Abschlussklausur - Völkerrecht: Geiselbefreiung im Ausland « in: Juristische Schulung 2004, S. 602 (603); Stephan Nicolaus Pieper, Völkerrechtliche Aspekte der internationalen Terrorismusbekämpfung, Diss., München 2004, S. 66.

14 Vgl. Thomas M. Franck, »Who killed Article 2 (4)? Or: Changing Norms Governing the Use of Force by States « in: American Journal of International Law 64 (1970), S. 809 ff.

15 Randelzhofer, »The Charter of the United Nations « aaO. (FN 10), Art. 2 Ziff. 4 Rdnr. 21.

16 Claus Kreß, »Strafrecht und Angriffskrieg im Licht des »Falles Irak«« in: Zeitschrift für die gesamte Strafrechtswissenschaft 115 (2003), S. 294 (299).

17 Dahm / Delbrück / Wolfrum, Völkerrecht, Bd. I/3, aaO. (FN 12), \$169 IX 4 FN 39 (S. 823); Bothe, Völkerrecht, aaO. (FN 10), 8. Abschnitt, Rdnr. 11; Christian Hillgruber, in: Jörg Menzel / Tobias Pierlings / Jeannine Hoffmann, (Hg.), Völkerrechtsprechung, Tübingen 2005, S. 815 f.

18 Schaller, »Das Friedenssicherungsrecht im Kampf gegen den Terrorismus« aaO. (FN 13), S. 9.

19 Fischer, Völkerrecht, aaO. (FN 10), $\ 59$ Rdnr. 20.

20 Kay Hailbronner, in: Dietrich Schindler / Kay Hailbronner, Die Grenzen des völkerrechtlichen Gewaltverbots, Berichte der Deutschen Gesellschaft für Völkerrecht, Heft 26, Heidelberg 1986, S. 67; Norbert K. Riedel, Der Einsatz deutscher Streitkräfte im Ausland - verfassungs- und völkerrechtliche Schranken, Frankfurt am Main u. a. 1989, S. 147. 
Recht zum - auch gewaltsamen - Schutz des eigenen befriedeten Territoriums gegen äußere Beeinträchtigungen aller Art, die ihrerseits nicht als »armed attack «i. S. des Art. 51 SVN zu qualifizieren sind, stützt sich auf die Gebietshoheit des betroffenen Staates.

Das völkerrechtliche Gewaltverbot umfasst deshalb grundsätzlich nicht die Abwehr gegen in das Staatsgebiet eindringende Personen, Flugzeuge oder Schiffe. ${ }^{21}$ In der Staatspraxis ist anerkannt, dass jedem Staat aus eigener Machtvollkommenheit kraft seiner territorialen Souveränität das Recht zusteht, gegen unerlaubt in den Luftraum eindringende Flugzeuge oder in Hoheitsgewässer eindringende Schiffe vorzugehen. ${ }^{22}$ Hiernach gehört insbesondere die Terrorismusbekämpfung in erster Linie zu den inneren Angelegenheiten eines Staates. Völkerrechtlich relevante Fragestellungen ergeben sich erst beim grenzüberschreitenden Terrorismus und seiner grenzüberschreitenden Bekämpfung. ${ }^{23}$

Politische und wirtschaftliche Gewalt, aber auch physische Gewalt, die wie bewaffnete Gewalt wirkt, werden nach überwiegender Meinung vom Gewaltverbot des Art. 2 Ziff. 4 SVN nicht erfasst. ${ }^{24}$

\section{Ausnabmen vom Gewaltverbot}

Ausnahmen vom Gewaltverbot sind die Zwangsmaßnahmen des Sicherheitsrats der VN und das Recht auf individuelle und kollektive Selbstverteidigung (Art. 51 SVN). Es wird darüber hinaus zunehmend die humanitäre Intervention als Ausnahme vom Gewaltverbot diskutiert. ${ }^{25}$

\section{a) Zwangsmaßnabmen des Sicherbeitsrats}

Dem Sicherheitsrat der VN ist nach Art. 24 Abs. 1 SVN die Hauptverantwortung für die Wahrung des Weltfriedens und der internationalen Sicherheit übertragen worden. Im Rahmen der friedlichen Beilegung von Streitigkeiten nach Kapitel VI SVN werden dem Sicherheitsrat durch die SVN Untersuchungs- und Empfehlungsrechte eingeräumt.

21 Alfred Verdross / Bruno Simma, Universelles Völkerrecht, 3. Aufl., Berlin 1984, S. 285 f.; Peter Wilkesmann, »Terroristische Angriffe auf die Sicherheit des Luftverkehrs in: Neue Zeitschrift für Verwaltungsrecht 2002, S. 1316 (1319).

22 Christian Tomuschat, »Der 11. September 2001 und seine rechtlichen Konsequenzen « in: Europäische Grundrechte-Zeitschrift 2001, S. 535 (540).

23 Dieter Blumenwitz, "Der Einsatz deutscher Streitkräfte nach der Entscheidung des BVerfG vom 12. Juli 1994« in: Bayerische Verwaltungsblätter 1986, S. 737.

24 Randelzhofer, »The Charter of the United Nations « aaO. (FN 10), Art. 2 Ziff. 4 Rdnr. 17 - 21; ders., in: Rudolf Bernhardt (Hg.), Encyclopedia Of Public International Law, Vol. 4, 2. Aufl., Amsterdam u. a. 2000, S. 1249.

25 Instruktiv: Hobe, »NATO-Intervention im Kosovo: Rückkehr zur Lehre vom gerechten Krieg?«aaO. (FN 6), S. 819 (822 ff.). 
Bei Friedensgefährdungen oder bei einem Friedensbruch muss der Sicherheitsrat vor einem Tätigwerden nach Kapitel VII SVN feststellen, ob eine Bedrohung oder ein Bruch des Friedens oder eine Angriffshandlung vorliegt (Art. 39 SVN).

Der Sicherheitsrat kann

- zur Einhaltung vorläufiger Maßnahmen auffordern,

- Empfehlungen abgeben oder

- nicht-militärische bzw. militärische Zwangsmaßnahmen anordnen.

Der Sicherheitsrat kann insbesondere nach Art. 42 SVN militärische Sanktionsmaßnahmen bestimmen. Der Sicherheitsrat kann aber auch die Mitgliedstaaten auffordern, selbst militärische Zwangsmaßnahmen zu ergreifen (Art. $48 \mathrm{SVN}) .{ }^{26}$ Der Sicherheitsrat ist schließlich befugt, den Mitgliedstaaten zu empfehlen oder sie aufzufordern, vom Recht der kollektiven Selbstverteidigung Gebrauch zu machen. Der Sicherheitsrat kann ein solches Recht in einem konkreten Fall auch anerkennen.

\section{b) Das Selbstverteidigungsrecht nach Art. S1 SVN}

Das Recht der individuellen und kollektiven Selbstverteidigung gilt unbestritten völkergewohnheitsrechtlich als allgemeine Regel des Völkerrechts ${ }^{27}$ und ist deshalb nach Art. 25 Satz 1 GG Bestandteil deutschen Bundesrechts. ${ }^{28}$

\section{Der bewaffnete Angriff}

Das Selbstverteidigungsrecht nach Art. 51 SVN $^{29}$ setzt einen »bewaffneten Angriff « (armed attack) auf einen Staat ${ }^{30}$ voraus. Der bewaffnete Angriff ist eine conditio sine

26 Vgl. Geiger, »Grundgesetz und Völkerrecht« aaO. (FN 5), \ 65 I 1 (S. 362).

27 Randelzhofer, »The Charter of the United Nations « aaO. (FN 10), Art. 51 Rdnr. 9 ff., 38.

28 Helmut Steinberger, in: Josef Isensee / Paul Kirchhof, (Hg.), Handbuch des Staatsrechts der Bundesrepublik Deutschland, Bd. VII, Heidelberg 1992, \ 173 Rdnr. 37 ff.; Paul Kirchhof, "Der Verteidigungsauftrag der deutschen Streitkräfte in: Ulrich Beyerlin, (Hg.), Recht zwischen Umbruch und Bewahrung: Völkerrecht, Europarecht, Staatsrecht; Festschrift für Rudolf Bernhardt, Berlin u. a. 1995, S. 797 (798).

29 Zur Diskussion, ob unter der VN-Charter weiterhin ein völkergewohnheitsrechtliches Selbstverteidigungsrecht existiert, instruktiv Christian von Buttlar, »Rechtsstreit oder Glaubensstreit?: Anmerkungen zur Debatte um die präventive Selbstverteidigung im Völkerrecht « in: Jürgen Bröhmer, (Hg.), Internationale Gemeinschaft und Menschenrechte, Festschrift für Georg Ress zum 70. Geburtstag am 21. Januar 2005, Köln/Berlin/ München 2005, S. 15 (16 ff.).

30 Stefan Talmon, »Grenzen der »Grenzenlosen Gerechtigkeit«. Die völkerrechtlichen Grenzen der Bekämpfung des internationalen Terrorismus nach dem 11. September 2001 « in: Wolfgang März, (Hg.), An den Grenzen des Rechts: Kolloquium zum 60. Geburtstag von Wolfgang Graf Vitzthum, 2003, S. 101 (149); Wiefelspütz, Das Parlamentsheer, Berlin 2005, S. 257. 
qua non für die Ausübung des Selbstverteidigungsrechts. ${ }^{31}$ Ein bewaffneter Angriff ist regelmäßig mit dem Einsatz militärischer Waffen verbunden. ${ }^{32}$ Diese Voraussetzung ist aber auch dann erfüllt, wenn an sich nicht-militärische Gegenstände wie beispielsweise Zivilflugzeuge zu Waffen umfunktioniert und zu Anschlägen missbraucht werden, die nach dem Ausmaß der Zerstörung und des Schadens einem herkömmlichen militärischen Angriff entsprechen. ${ }^{33}$

Nicht jede Gewaltanwendung ist bereits ein bewaffneter Angriff. Ein bewaffneter Angriff liegt nach vorherrschender Auffassung in der völkerrechtlichen Literatur nur vor, wenn Gewalt in größerem Umfang angewendet wird. ${ }^{34}$

Angriffe auf staatliche Außenposten können unter Umständen einem bewaffneten Angriff i. S. des Art. 51 SVN gleichkommen. Angriffe auf die Land-, See- und Luftstreitkräfte, auf Militärstützpunkte im Ausland sowie die Handelsmarine oder die zivile Luftflotte eines Staates werden völkerrechtlich wie der bewaffnete Angriff auf den Heimatstaat bewertet, wenn diese Operationen von einigem Gewicht sind. ${ }^{35}$

Angriffe auf einzelne Staatsangehörige sind in der Regel nicht als bewaffneter Angriff i. S. des Art. 51 SVN anzusehen. ${ }^{36}$ Ein Staat wird nämlich durch Angriffe auf Staatsangehörige im Ausland regelmäßig nicht in seiner Integrität oder Unabhängigkeit bedroht. Anderes kann aber dann gelten, wenn Menschen in größerer Zahl wegen ihrer Staatsangehörigkeit angegriffen werden. Rettungsaktionen kommen freilich als humanitäre Intervention und damit als Ausnahme vom Gewaltverbot in Betracht. ${ }^{37}$

31 Nicaragua, ICJ Reports 1986, S. 14 (122, Ziff. 236 f.); IGH, Case concerning Oil Platforms (Islamic Republic of Iran v. United States of America), Merits, Urteil vom 6. November 2003, \51, www.icj-cij.org; Eva-Maria Schulze, "Selbstverteidigung « in: Rüdiger Wolfrum (Hg.), Handbuch der Vereinten Nationen, 2. Aufl., München 1991, (102) Rdnr. 3 (S. 753); Christian Schaller, »Massenvernichtungswaffen und Präventivkrieg - Möglichkeiten der Rechtfertigung einer militärischen Intervention im Irak aus völkerrechtlicher Sicht « in: Zeitschrift für ausländisches öffentliches Recht und Völkerrecht 62 (2002), S. 641 (657 f.).

32 Dörr, »Gewalt und Gewaltverbot im modernen Völkerrecht«, aaO. (FN 5), S. 14 (15).

33 Dederer, »Krieg gegen Terror «, aaO. (FN 13), S. 421 (424 f.).

34 Martin Limpert, Auslandseinsatz der Bundeswehr, Berlin 2002, S. 41; Fischer, Völkerrecht, aaO. (FN 10), $\ 59$ Rdnr. 28; Klaus Kersting, Bündnisfall und Verteidigungsfall, Diss., Bochum 1979, S. 79; kritisch: Karl Doehring, Völkerrecht, 2. Aufl., Heidelberg 2004, Rdnr. 761 ff.; Wolff Heintschel von Heinegg / Tobias Gries, »Der Einsatz der Deutschen Marine im Rahmen der Operation »Enduring Freedom« in: Archiv des Völkerrechts 2002, S. 145 (153); Rudolf Streinz, »Wo steht das Gewaltverbot heute? « in: Jabrbuch des öffentlichen Rechts der Gegenwart N.F. 52 (2004), S. 219 (224.

35 Pieper, »Völkerrechtliche Aspekte der internationalen Terrorismusbekämpfung « aaO. (FN 13), S. 105.

36 Randelzhofer, »The Charter of the United Nations « aaO. (FN 10), Art. 51 Rdnr. 26 m. w. N.; Carsten Stahn, »'Nicaragua is dead, long live Nicaragua' - the Right to Selfdefence Under Art. 51 UN-Charter and International Terrorism« in: Christian Walter / Silja Vöneky / Volker Röben / Frank Schorkopf, Hg.), in: Terrorism as a Challenge for National and International Law: Security versus Liberty?, Berlin u. a. 2004, S. 827 (833).

37 Stein / von Buttlar, Völkerrecht, aaO. (FN 1), Rdnr. 805 (»ungeschriebener Rechtfertigungsgrund «); Herdegen, Völkerrecht, aaO. (FN 4), Rdnr. 21 f. 
Meinungen, die unterhalb der Angriffsschwelle eine »kleine Selbstverteidigung « für zulässig halten, sind in der Minderheit geblieben. ${ }^{38}$ So sind insbesondere Grenzzwischenfälle zwar ein Verstoß gegen das Gewaltverbot des Art. 2 Ziff. 4 SVN, nicht aber ein bewaffneter Angriff. Der durch solche Gewaltakte verletzte Staat ist gleichwohl nicht rechtlos. Fraglos ist ein Staat berechtigt, jede rechtswidrige Verletzung seines Territoriums - gegebenenfalls auch mit militärischen Mitteln - abzuwehren. ${ }^{39}$ Auch Gewaltakte von geringerer Bedeutung verletzen nämlich die Gebietshoheit eines Staates. Sie rechtfertigen freilich (nur) Maßnahmen zur Abwehr des konkreten Angriffs. Dies darf allerdings - wenn erforderlich - auch mit militärischen Mitteln erfolgen.

Die Generalversammlung der VN unternahm mit der Resolution 3314 (XXIX) vom 14. Dezember 1974 den Versuch, den Begriff der Aggression näher zu umschreiben. Auf diese Resolution wird regelmäßig zurückgegriffen, um zu beurteilen, ob es sich bei der Anwendung von Gewalt um einen bewaffneten Angriff handelt. ${ }^{40}$ Danach spricht beim Ersteinsatz militärischer Gewalt eine Vermutung dafür, dass ein Akt der Aggression vorliegt, es sei denn, der Sicherheitsrat der VN kommt zu einer anderen Bewertung.

Anders als die Wahrnehmung der Gebietshoheit umfasst das Selbstverteidigungsrecht nach Art. 51 SVN nicht nur das Recht auf militärische Abwehr des Angreifers an der Grenze, sondern auch das Recht, Streitkräfte über die Grenze hinaus ins Ausland zu entsenden, um dort alle Maßnahmen zu ergreifen, die für eine wirksame Verteidigung notwendig sind.

Der bewaffnete Angriff muss noch gegenwärtig sein. ${ }^{41}$ Deshalb müssen Selbstverteidigungsmaßnahmen unmittelbar dem bewaffneten Angriff folgen. ${ }^{42}$ Außerdem müssen sie im Hinblick auf den bewaffneten Angriff verhältnismäßig sein. ${ }^{43}$ Bei einem singulären, abgeschlossenen terroristischen Anschlag erlischt das Recht auf Selbstverteidigung, selbst wenn der Anschlag als bewaffneter Angriff i. S. des Art. 51 SVN gewertet werden durfte. ${ }^{44}$ Zur typischen Struktur terroristischer Umtriebe gehört freilich, dass die Täter zunächst untertauchen und erst nach geraumer Zeit an anderer Stelle erneut Anschläge verüben. ${ }^{45}$ Man wird deshalb das Erfordernis der

38 Vgl. Dörr, »Das völkerrechtliche Gewaltverbot am Beginn des 21. Jahrhunderts - Was bleibt von Art. 2 (4) UN-Charta?«aaO. (FN 13), S. 33 (38).

39 Vgl. Herdegen, Völkerrecht, aaO. (FN 4), \$34 Rdnr. 7.

40 Dieter Blumenwitz, »Einsatzmöglichkeiten der Bundeswehr im Kampf gegen den Terrorismus « in: Zeitschrift für Rechtspolitik 2002, S. 102 (104).

41 Thomas Bruha / Matthias Bortfeld, "Terrorismus und Selbstverteidigung « in: Vereinte Nationen 2001, S. 161 (165).

42 Kugelmann, »Die völkerrechtliche Zulässigkeit von Gewalt gegen Terroristen « in: Juristische Ausbildung 2003, S. 376 (381).

43 Fischer, Völkerrecht, aaO. (FN 10), \59 Rdnr. 39; Jochen Abr. Frowein, »Der Terrorismus als Herausforderung für das Völkerrecht « in: Zeitschrift für ausländisches öffentliches Recht und Völkerrecht 62 (2002), S. 879 (888).

44 Bruha / Bortfeld, Terrorismus und Selbstverteidigung, aaO. (FN 41), S. 161 (165); vgl. aber Herdegen, Völkerrecht, aaO. (FN 4), $\$ 34$ Rdnr. 16.

45 Vgl. Claus Kreß, »Jus contra Bellum: Quo vadis? « in: Kursbuch Heft 155 (2004), S. 62 (77). 
Gegenwärtigkeit des Angriffs bei terroristischen Anschlägen weiter fassen müssen. ${ }^{46}$ Das Recht auf Selbstverteidigung darf nicht dadurch geschmälert werden, dass der angegriffene Staat die terroristischen Strukturen und Verantwortlichkeiten zunächst klären muss. ${ }^{47}$ Dem angegriffenen Staat wird deshalb ein gewisser Ermessensspielraum bei der Art und Weise der Verteidigungsmaßnahmen einzuräumen sein..$^{4}$ Dem angegriffenen Staat wird auch ein angemessener Zeitraum zur Planung und Vorbereitung der militärische Verteidigungshandlungen zu gewähren sein.

\section{Nichtstaatliche Gewalt}

Die zunehmende Privatisierung der Gewalt bei bewaffneten Konflikten und die typischen Formen des internationalen Terrorismus werfen zunehmend die Fragestellung auf, ob ein »bewaffneter Angriff « die Verstrickung eines Staates voraussetzt. ${ }^{49}$

Es bedarf auch der Klärung, ob der angegriffene Staat im Rahmen der Selbstverteidigung terroristische Stützpunkte oder Basen im jeweiligen Aufenthaltsstaat bekämpfen darf. In demselben Zusammenhang fragt sich, unter welchen Voraussetzungen der Aufnahmestaat selber als Angreifer zu qualifizieren ist.

\section{a) Staatliche und nichtstaatliche Gewalt}

Insbesondere die terroristischen Anschläge vom 11. September 2001 in den USA haben zu einer Neubewertung der Voraussetzungen für einen bewaffneten Angriff i. S. des Art. 51 SVN geführt. ${ }^{50}$ Terroristische Anschläge wurden bislang lediglich als

46 Delbrück, »The Fight Against Global Terrorism: Self-Defense or Collective Security as International Police Action? Some Comments on the International Legal Implications of the 'War Against Terrorism' « aaO (FN 7), S. 9 (16); Hanspeter Neuhold, »Law and Force in International Relations - European and European and American Positions « in: Zeitschrift für ausländisches öffentliches Recht und Völkerrecht 64 (2004), S. 263 (272 f.).

47 Vgl. Roman Schmidt-Radefeldt, »Parlamentary Accountability and Military Forces in NATO: The Case of Germany « in: Hans Born / Heiner Hänggi, (Hg.), The ,Double Democratic Deficit'. Parliamentary Accountability and the Use of Force Under International Auspices, Aldershot u. a. 2004, S. 147 (156).

48 Tomuschat, »Der 11. September 2001 und seine rechtlichen Konsequenzen« aaO. (FN 22), S. 535 (542).

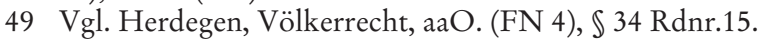

50 Instruktiv: Hillgenberg, »Gewaltverbot: Was gilt noch?« aaO. (FN 8), S. 141 (153 ff.); Kreß, »Jus contra Bellum: Quo vadis? « aaO. (FN 45), S. 62 (65 ff.). Die Versuche, eine umfassende Terrorismuskonvention zu schaffen, sind freilich bis heute gescheitert. Vgl. dazu Fischer, Völkerrecht, aaO. (FN 10), \59 Rdnr. 13; Thomas Bruha, »Gewaltverbot und humanitäres Völkerrecht nach dem 11. September 2001 « in: Archiv des Völkerrechts 40 (2002), S. 383 (393 ff.); Michael N. Schmitt, »Preemptive Strategies in International Law « in: Michigan Journal of International Law Vol. 24 (2003), S. 513 (536 ff.); Christophe Eick, »'Präemption', ,Prävention' und die Weiterentwicklung des Völkerrechts « in: Zeitschrift für Rechtspolitik 2004, S. 200; Eckart Klein, »Die Herausforderung durch den internationalen Terrorismus: hört hier das Völkerrecht auf? « in: Josef Isensee, (Hg.), Der Terror, der Staat und das Recht, 2004, S. 9 (23 ff.). 
schwere Straftaten angesehen, die im Rahmen internationaler Konventionen als Straftaten nach nationalem Recht und nach dem überkommenen Grundsatz des Völkerrechts »dedere aut judicare « behandelt wurden. ${ }^{51}$ Dies soll hier nicht in Frage gestellt werden..$^{52}$ Terroristen sind Schwerstverbrecher, nicht aber Kombattanten im Sinne des Kriegsrechts. ${ }^{53}$ Die Grenze zwischen Strafrecht und Völkerrecht darf auch in Zukunft nicht verwischt werden. ${ }^{54}$ Selbst wenn terroristische Anschläge militärische Dimensionen erreichen können, macht es wenig Sinn, aus Verbrechern Kombattanten zu machen.

Es setzt sich freilich zunehmend die Erkenntnis durch, dass allein eine ausschließlich strafrechtliche Sanktionierung terroristischer Umtriebe keine angemessene und ausreichende Reaktion ist. Die terroristischen Anschläge vom 11. September 2001 haben gezeigt, dass Terrorismus auch zu einer unmittelbaren Gefährdung des Weltfriedens und der internationalen Sicherheit führen kann..$^{55}$ Gleichwohl sah der Sicherheitsrat der VN bislang davon ab, militärische Zwangsmaßnahmen gegen terroristische Organisationen zu autorisieren. Der Sicherheitsrat beschränkte sich darauf, das naturgegebene Recht zur individuellen und kollektiven Selbstverteidigung anzuerkennen und zu bekräftigen. ${ }^{56}$

Unstreitig ist, dass die Unterstützung, ja bereits die Tolerierung terroristischer Aktionen, die sich gegen einen Staat richten, völkerrechtswidrig ist. Sind der bewaffnete Angriff und die Steuerung der Tat ausschließlich auf dem Territorium des angegriffenen Staates zu lokalisieren, ist nicht das Völkerrecht, sondern das nationale Recht einschlägig. Wird ein Staat von einer terroristischen Organisation angegriffen, die auf der Hohen See oder in einem Flugzeug über der Hohen See operiert, kann der angegriffene Staat militärische Gegenmaßnahmen ergreifen, ohne in Konflikt mit dem Gewaltverbot des Art. 2 Ziff. 4 SVN zu kommen. ${ }^{57}$ In diesem Fall darf sich der angegriffene Staat auf seine unbeschränkte Souveränität berufen.

51 Vgl. Hillgenberg, »Gewaltverbot: Was gilt noch?«aaO. (FN 8), S. 141 (154); Christian Tomuschat, »Präventivkrieg zur Bekämpfung des internationalen Terrorismus?« in: Deutschen Institut für Menschenrechte / Gabriele von Arnim / Volkmar Deile / FranzJosef Hutter / Sabine Kurtenbach / Carsten Tessmer, (Hg.), Jabrbuch Menschenrechte 2004, Frankfurt am Main 2003, S. 121 (124).

52 Zur »strafrechtlichen Lösung «vgl. Christian Walter, »Zwischen Selbstverteidigung und Völkerstrafrecht: Bausteine für ein internationales Recht der,präventiven TerrorismusBekämpfung' « in: Dieter Fleck, (Hg.), Rechtsfragen der Terrorismusbekämpfung durch Streitkräfte, Baden-Baden 2004, S. 23 (31).

53 Dieter Blumenwitz, »Die völkerrechtlichen Aspekte des Irak-Konflikts « in: Zeitschrift für Politik 50 (2003), S. 301 (312).

54 Vgl. Hans-Joachim Heintze, »Das Völkerrecht wird unterschätzt: internationale Antworten auf den internationalen Terrorismus « in: Internationale Politik und Gesellschaft 3/2004, S. 38 (44 ff.).

55 Dahm / Delbrück / Rüdiger Wolfrum, Völkerrecht, Bd. I/2, Der Staat und andere Völkerrechtssubjekte; Räume und internationale Verwaltung, Berlin u. a. O. 2002, \} 1 9 5 \text { II } 1 (S. 1112).

56 SR-Res. 1368 (2001); 1373 (2001).

57 Tomuschat, »Präventivkrieg zur Bekämpfung des internationalen Terrorismus?« aaO. (FN 51), S. 121 (127). 
Traditionell wurde davon ausgegangen, dass ein »bewaffneter Angriff « nur von einem Staat geführt werden kann. ${ }^{58}$ Dementsprechend wurden nichtstaatliche terroristische Anschläge nicht als »bewaffneten Angriffe « gewertet, gegen die ein Staat das Selbstverteidigungsrecht nach Art. 51 SVN in Anspruch nehmen darf. ${ }^{59}$ Diese Auffassung war bis zu den Anschlägen vom 11. September 2001 vorherrschend. ${ }^{60}$

Der Wortlaut des Art. 51 SVN rechtfertigt freilich eine solche Interpretation nicht, denn es ist lediglich die Rede von »armed attack «, nicht von »armed attack by a state « ${ }^{61}$ Ein bewaffneter Angriff kann aber vom Territorium eines anderen Staates ausgehen. Entscheiden ist, dass er von außen gesteuert wird. ${ }^{62}$

Inzwischen wird immer häufiger die Meinung vertreten, dass ein »bewaffneter Angriff « auch von einer nichtstaatlichen terroristischen Organisation ausgehen kann, wenn ein Staat ihr durch bloße Duldung auf seinem Staatsgebiet Operationsmöglichkeiten einräumt. ${ }^{63}$ Gewalt von nichtstaatlichen Gruppen sei dann als bewaffneter Angriff zu werten ist, wenn die Gewalt kriegsmäßiges Ausmaß erreiche. ${ }^{64}$ Entscheidend sei, dass die Angriffe nicht vom Territorium des Zielstaates ihren Ausgang nähmen und ihre Durchführung nur dadurch möglich gewesen sei, dass das hinter den Terroristen stehende Netzwerk transnationale Züge trage. Allgemein sei festzustellen, dass es einem angegriffenen Staat möglich sein müsse, unter Wahrung der territorialen Souveränität des Drittstaates eine effektive Verfolgung der terroristischen Organisationen vorzunehmen. ${ }^{65}$

58 Vgl. Schulze, »Selbstverteidigung «aaO. (FN 31), (102) Rdnr. 17 (756 f.); Randelzhofer, »The Charter of the United Nations « aaO. (FN 10), Art. 51 Rdnr. 35; Gerhard Zimmer, Terrorismus und Völkerrecht: militärische Zwangsanwendung, Selbstverteidigung und Schutz der internationalen Sicherbeit, Aachen 1998, S. 56; Christian Tomuschat, "Völkerrechtliche Aspekte bewaffneter Konflikte« in: Leviathan 2003, S. 450 (458).

59 Randelzhofer, »The Charter of the United Nations «, aaO. (FN 10), Art. 51 Rdnr. 34; Jost Delbrück, »Effektivität des UN-Gewaltverbots. Bedarf es einer Modifikation der Reichweite des Art. 2 (4) UN-Charta? in: Die Friedens-Warte 1999, S. 139 (156); Christian Tietje / Karsten Nowrot, »Völkerrechtliche Aspekte militärischer Maßnahmen gegen den internationalen Terrorismus « in: Neue Zeitschrift für Webrrecht 2002, S. 1 (5 f.); Markus Krajewski, »Das Parlamentsheer als Kollateralschaden der Irak-Krieges? « in: Archiv des Völkerrechts 40 (2002), S. 183 (187 f.).

60 Vgl. Claus Kreß, Gewaltverbot und Selbstverteidigungsrecht nach der Satzung der Vereinten Nationen bei staatlicher Verwicklung in Gewaltakte Privater, Berlin 1995, S. 206 m. w. N.

61 Stahn, "'Nicaragua is dead, long live Nicaragua' - the Right to Self-defence Under Art. $51 \mathrm{UN}-\mathrm{Charter}$ and International Terrorism«, aaO. (FN 36), S. 827 (830, 848 f.).

62 Vgl. Stahn, »'Nicaragua is dead, long live Nicaragua' - the Right to Self-defence Under Art. 51 UN-Charter and International Terrorism«, aaO. (FN 36), S. 827 (849); Blumenwitz, »Die völkerrechtlichen Aspekte des Irak-Konflikts « aaO. (FN 53), S. 301 (312).

63 Herdegen, Völkerrecht, aaO. (FN 4), \34 Rdnr.15; Streinz, »Wo steht das Gewaltverbot heute?« aaO. (FN 34), S. 219 (225); Volker Böge, »'Landesverteidigung' am Hindukusch« in: Till Müller-Heidelberg, Ulrich Finckh, Elke Steven, Bela Rogalla, Jürgen Micksch, Wolfgang Kaleck, Martin Kutscha, (Hg.), Grundrechte-Report 2003, Frankfurt am Main 2003, S. 173 (175 f.).

64 Vgl. Randelzhofer, »The Charter of the United Nations « aaO. (FN 10), Art. 51 Rdnr. 34; Hillgenberg, »Gewaltverbot: Was gilt noch?«aaO. (FN 8), S. 141 (154).

65 Heintschel von Heinegg / Gries, »Der Einsatz der Deutschen Marine im Rahmen der Operation ,Enduring Freedom”", aaO. (FN 34), S. 145 (156 ff.); Pieper, »Völkerrechtliche Aspekte der internationalen Terrorismusbekämpfung «, aaO. (FN 13), S. 91. 
Mittlerweile scheint sich die Auffassung durchzusetzen, dass es zur Feststellung der Selbstverteidigungssituation eines Staates keines staatlichen Zurechnungssubjekts für die Angriffshandlung bedarf, ${ }^{66}$ was auch der Staatenpraxis entspricht. ${ }^{67}$ Die Zurechnung eines Anschlages sei nicht für die Definition eines bewaffneten Angriffs von Bedeutung, sondern für die Auswahl des Adressaten der Verteidigungsmaßnahmen. ${ }^{68}$ In einer Zeit, in der terroristische Organisationen über Massenvernichtungswaffen zu verfügen imstande seien oder in der Lage seien, Anschläge zu verüben, die in ihrer Zerstörungskraft herkömmlichen militärischen Operationen nahe kommen oder diese gar übertreffen, könne die Berechtigung zur Selbstverteidigung nicht davon abhängen, ob ein Staat dahinter stehe oder nicht. ${ }^{69}$ Der Begriff des bewaffneten Angriffs sei ausschließlich materiell zu verstehen. Eine Verknüpfung zwischen privater Gewaltanwendung und staatlicher Verantwortung sei nicht erforderlich. ${ }^{70}$

Vereinzelt wird erwogen, terroristischen Organisationen partielle Völkerrechtssubjektivität zuzusprechen ${ }^{71}$ es wird auch von einem völkerrechtlichen Rechtsverhältnis zwischen dem angegriffenen Staat und der terroristischen Organisation ausgegangen. ${ }^{72}$

Verallgemeinernd lässt sich heute feststellen: Ein terroristischer Anschlag wird inzwischen jedenfalls dann als bewaffneter Angriff gewertet, wenn er mit Waffengewalt erfolgt und ein Ausmaß erreicht, das nach Umfang und Auswirkungen dem Angriff regulärer Streitkräfte entspricht. Demgegenüber wird von Gerd Seidel eingewandt, Art. 51 SVN stelle auf ein zwischenstaatliches Verhältnis zwischen Angreifer und Angegriffenem ab. Es habe sich bislang weder eine völkerrechtliche Praxis noch die erforderliche opinio juris herausgebildet, die eine Änderung des Initiators eines bewaffneten Angriffs bewirkt haben könnte. ${ }^{73}$ Die Anerkennung

66 Schulze, »Selbstverteidigung « aaO. (FN 31), (102) Rdnr. 19 (S. 757); Matthias Ruffert, »Terrorismusbekämpfung zwischen Selbstverteidigung und kollektiver Sicherheit: die Anschläge vom 11.9.2001 und die Intervention in Afghanistan « in: Zeitschrift für Rechtspolitik 2002, S. 247 f.; Sean D. Murphy, »Terrorism and the Concept of Armed Attack in Article 51 on the U.N. Charter in: Harvard International Law Journal 43 (2002), S. 41 (50); Klein, »Herausforderung durch den internationalen Terrorismus: hört hier das Völkerrecht auf? «aaO. (FN 50), S. 9 (27 ff.).

67 Schmitt, »Preemptive Strategies in International Law «aaO. (FN 50), S. 513 (536).

68 Walter, »Zwischen Selbstverteidigung und Völkerstrafrecht: Bausteine für ein internationales Recht der »präventiven Terrorismus-Bekämpfung «aaO. (FN 52), S. 23 (26).

69 Frowein, »Der Terrorismus als Herausforderung für das Völkerrecht« aaO. (FN 43), S. 879 (887).

70 Bruha, »Gewaltverbot und humanitäres Völkerrecht nach dem 11. September 2001 « aaO. (FN 50), S. 383 (395 m. w. N. in FN 62).

71 Delbrück, »Effektivität des UN-Gewaltverbots. Bedarf es einer Modifikation der Reichweite des Art. 2 (4) UN-Charta?«aaO. (FN 59), S. 139 (156).

72 Vgl. Bruha / Bortfeld, » Terrorismus und Selbstverteidigung «aaO. (FN 41), S. 161 (163); wohl auch Krajewski, »Das Parlamentsheer als Kollateralschaden der Irak-Krieges? « aaO. (FN 59), S. 183 (202); ablehnend Talmon, »Grenzen der ,Grenzenlosen Gerechtigkeit'. Die völkerrechtlichen Grenzen der Bekämpfung des internationalen Terrorismus nach dem 11. September 2001《 aaO. (FN 30), S. 101 (168).

73 Gerd Seidel, "Quo vadis Völkerrecht? « in: Archiv des Völkerrechts 41 (2003), S. 449 (461); ähnlich argumentiert Hillgruber, »Völkerrechtsprechung «aaO. (FN 17), S. 820 f. 
terroristischer Organisationen als partielle Völkerrechtssubjekte hieße, dass künftig der von einem bewaffneten Angriff betroffene Staat einen beliebigen Staat, in dem sich Terroristen vermeintlich oder tatsächlich niedergelassen haben, als Zielstaat einer Selbstverteidigungsmaßnahme auswählen könnte. Damit könnte das Selbstverteidigungsrecht entgegen der ratio des Art. 51 SVN in inflationärem Umfang ausgedehnt werden. ${ }^{74}$

Die Auffassung von Gerd Seidel überzeugt nicht. Aus der Sicht des angegriffenen Staates ist es unerheblich, ob die Gewalt von einem Staat oder einer anderen Organisation ausgeht. Der Wortlaut des Art. 51 SVN enthält kein »präzisierendes « staatliches Tatbestandsmerkmal. ${ }^{75}$ Für die Annahme eines bewaffneten Angriffs im Sinne des Art. 51 SVN ist entscheidend, dass ein Staat Opfer militärische Gewalt von größerem Umfang ist. Die Beschränkung des Selbstverteidigungsrechts auf staatliche Angriffe würde der Schutzfunktion des Art. 51 SVN nicht gerecht. Die Fixierung auf staatliche Angriffe würde im Falle von (nichtstaatlichen) terroristischen Anschlägen von einem Gewicht und einer Tragweite, die mit einem erheblichen militärischen Angriff vergleichbar sind, eine nicht verantwortbare Schutzlücke entstehen lassen. Deshalb kommen auch nichtstaatliche Gruppen als Gegner der staatlichen Selbstverteidigung nach Art. 51 SVN in Betracht. ${ }^{76}$ Das Vorliegen eines bewaffneten Angriffs löst das Selbstverteidigungsrecht gegenüber der privaten, nichtstaatlichen oder transnationalen Organisation aus. Dies ermächtigt zu militärischen Maßnahmen gegen diese Gruppierung. Das schließt deren Bekämpfung in hoheitsfreien Räumen (Luftraum, Hohe See) ein.. ${ }^{77}$

\section{b) Die Zurechnung eines bewaffneten Angriffs}

Im Falle eines bewaffneten Angriffs durch eine nichtstaatliche Gruppe sind die Verteidigungsmaßnahmen grundsätzlich nur gegen diese Gruppe und nicht gegen den Aufenthaltsstaat zu richten. ${ }^{78}$ Alle Staaten sind verpflichtet, den internationalen Terrorismus zu bekämpfen und terroristische Akte zu verhindern, die von ihrem Hoheitsgebiet ausgehen und gegen Ziele in anderen Staaten gerichtet sind..$^{79}$ Kommt ein Staat dieser Verpflichtung nicht nach, darf sich der Aufenthaltsstaat nicht uneingeschränkt auf seine territoriale Unversehrtheit berufen und muss in begrenztem Um-

74 Seidel, »Quo vadis Völkerrecht?« aaO. (FN 73), S. 449 (462); vgl. auch Antonio Cassese, »Terrorism is also disrupting some crucial legal categories of international law « in: European journal of international law 12 (2001), S. 993 (997).

75 Tomuschat, »Völkerrechtliche Aspekte bewaffneter Konflikte« aaO. (FN 58), S. 450 (458).

76 Hillgenberg, »Gewaltverbot: Was gilt noch?«aaO. (FN 8), S. 141 (154).

77 Bruha / Bortfeld, »Terrorismus und Selbstverteidigung « aaO. (FN 41), S. 161 (166).

78 Schaller, »Das Friedenssicherungsrecht im Kampf gegen den Terrorismus «aaO. (FN 13), S. 14.

79 Frowein, »Der Terrorismus als Herausforderung für das Völkerrecht« aaO. (FN 43), S. 879 (883); Heintze, »Das Völkerrecht wird unterschätzt: internationale Antworten auf den internationalen Terrorismus« aaO. (FN 54), S. 38 (41 ff.). 
fang Verteidigungsmaßnahmen des angegriffenen Staates auf seinem Territorium dulden. ${ }^{80}$ Denn das Selbstverteidigungsrecht des angegriffenen Staates überwiegt das territoriale Interesse des angegriffenen Staates. Der angegriffene Staat darf in einem solchen Fall die Maßnahmen ergreifen, die der Aufenthaltsstaat hätte ergreifen müssen. ${ }^{81}$ Weitere Voraussetzung ist aber stets, dass der Anschlag auf den angegriffenen Staat das Ausmaß eines bewaffneten Angriffs i. S. des Art. 51 SVN erreicht. ${ }^{82}$

Aber auch wenn ein Aufenthaltsstaat aufgrund eigener Zerfallserscheinungen eine terroristische Organisation nicht effektiv zu bekämpfen in der Lage ist, muss er gewaltsame Maßnahmen der Selbstverteidigung auf seinem Staatsgebiet dulden..$^{83}$ Voraussetzung für einen Einsatz auf fremdem Territorium ist allerdings immer, dass mit hoher Plausibilität die Gefährdung des eigenen Territoriums durch terroristische Aktionen nachgewiesen werden kann.

Ein Selbstverteidigungsfall liegt auch dann vor, wenn die terroristischen Täter auf dem Territorium des angegriffenen Staates leben, wenn der Anschlag von einem fremden Staat oder einer fremden, nicht im Zielstaat ansässigen Organisation kontrolliert wird. ${ }^{84} \mathrm{Ob}$ freilich gegen den Aufnahmestaat selbst als Objekt des Selbstverteidigungsrechts militärisch vorgegangen werden darf, ist davon abhängig, ob die Gewalttaten diesem Staat zugerechnet werden können. ${ }^{85}$ Wenn ein Staat Terroristen entsendet, um einen anderen Staat anzugreifen, handeln die Terroristen - de jure oder de facto - als Organe des den Anschlag initiierenden Staates. ${ }^{86}$

Die Tatsache, dass eine nichtstaatliche terroristische Gruppe vom Staatsgebiet des Aufenthaltsstaates aus operiert, genügt allein nicht, die Gewalttaten dem Aufenthaltsstaat zuzurechnen. ${ }^{87}$ In seinem Nicaragua-Urteil ${ }^{88}$ ging der IGH davon aus,

80 Schmitt, »Preemptive Strategies in International Law «aaO. (FN 50), S. 513 (540 ff.); Tomuschat, »Präventivkrieg zur Bekämpfung des internationalen Terrorismus?« aaO. (FN 51), S. 121 (127).

81 Dinstein, »Comments on the Presentations by Nico Kirsch and Carsten Stahn« aaO. (FN 8), S. 915 (922 f.) spricht in diesem Fall von »extra-territorial law enforcement«.

82 Walter, "Zwischen Selbstverteidigung und Völkerstrafrecht: Bausteine für ein internationales Recht der »präventiven Terrorismus-Bekämpfung « aaO. (FN 52), S. 23 (41).

83 Randelzhofer, »The Charter of the United Nations « aaO. (FN 10), Art. 51 Rdnr. 36; Herdegen, Völkerrecht, aaO. (FN 4), \34 Rdnr.15; Jochen Abr. Frowein, »Ist das Völkerrecht tot? in: Frankfurter Allgemeine Zeitung vom 23. Juli 2003.

84 Frowein, »Der Terrorismus als Herausforderung für das Völkerrecht « aaO. (FN 43), S. 879 (886).

85 Vgl. Schulze, »Selbstverteidigung «aaO. (FN 31), (102) Rdnr. 17 ff. (S. 756 f); instruktiv die Übersicht bei Dörr, »Das völkerrechtliche Gewaltverbot am Beginn des 21. Jahrhunderts - Was bleibt von Art. 2 (4) UN-Charta?«aaO. (FN 13), S. 33 (39 ff.); Kugelmann, »Die völkerrechtliche Zulässigkeit von Gewalt gegen Terroristen « aaO. (FN 42), S. 376 (378 f.).

86 Vgl. Art. 3 lit g General Assembly Resolution 3314 (XXIX); siehe auch Dinstein, »Comments on the Presentations by Nico Kirsch and Carsten Stahn« aaO. (FN 8), S. 915 (919); Blumenwitz, »Die völkerrechtlichen Aspekte des Irak-Konflikts« aaO. (FN 53), S. 301 (312 f.).

87 Vgl. Seidel, »Quo vadis Völkerrecht?« aaO. (FN 73), S. 449 (463 ff.); Schaller, »Das Friedenssicherungsrecht im Kampf gegen den Terrorismus «aaO. (FN 13), S. 17.

88 IGH Reports 1986, S. 14 et seq., 103-123, Ziff. 195. 
dass die Entsendung bewaffneter Banden, nicht aber bereits die bloße Unterstützung dieser Banden einen bewaffneten Angriff darstellt. ${ }^{89}$ Der Aufenthaltsstaat musste gleichsam »Tatherrschaft « über die terroristischen Aktionen haben. ${ }^{90}$ Die ICL forderte noch im August 2001 »direction or control « für die Zurechenbarkeit nichtstaatlichen Handelns. ${ }^{91}$

Mittlerweile scheint sich insbesondere in unmittelbarer Reaktion auf die Anschläge vom 11. September 2001 die Auffassung durchgesetzt zu haben, dass bei der Zurechnung erweiternde Kriterien ausreichen. ${ }^{92}$ Auch derjenige Staat gilt als legitimes Ziel der Selbstverteidigung, der private Gewalttäter im weiteren Sinne unterstützt. ${ }^{93}$ Die Unterstützung einer terroristischen Gruppierung, aber auch das Schützen oder zumindest wissentliche Dulden (»harboring «) solcher Gruppen auf eigenem Hoheitsgebiet genügt für eine Zurechnung. ${ }^{94}$ Nicht erforderlich ist, dass die terroristische Gruppierung vom Aufenthaltsstaat gesteuert wird. ${ }^{95}$ Der Verdacht einer Unterstützung reicht freilich nicht aus, um gegen diesen Staat militärisch vorgehen zu dürfen.

Die Voraussetzungen des Art. 51 SVN können auch dann gegeben sein, wenn Terroristen von einem Aufenthaltsstaat aus einen anderen Staat angreifen, und der Anschlag erst nachträglich vom Aufenthaltsstaat gebilligt wird. Auch in diesem Fall werden die Terroristen faktisch zu Organen des Aufenthaltsstaates. Wenn der bewaffnete Angriff noch andauert, kann der angegriffene Staat im Rahmen seines Rechts auf Selbstverteidigung auch gegen den Aufenthaltsstaat militärisch vorgehen. ${ }^{96}$

89 Vgl. auch Schaller, »Das Friedenssicherungsrecht im Kampf gegen den Terrorismus« aaO. (FN 13), S. 16.

90 Blumenwitz, »Einsatzmöglichkeiten der Bundeswehr im Kampf gegen den Terrorismus « aaO. (FN 40), S. 102 (104); Thomas Bruha, »Neuer Internationaler Terrorismus: Völkerrecht im Wandel? « in: Hans-Joachim Koch, (Hg.), Terrorismus - Rechtsfragen der äußeren und inneren Sicherheit, Baden-Baden 2002, S. 51 (67).

91 Hillgenberg, »Gewaltverbot: Was gilt noch?« aaO. (FN 8), S. 141 (155).

92 Schaller, »Das Friedenssicherungsrecht im Kampf gegen den Terrorismus « aaO. (FN 13), S. 16 f.; Hillgruber, »Völkerrechtsprechung « aaO. (FN 17), S. 815 f.

93 Randelzhofer, »The Charter of the United Nations « aaO. (FN 10), Art. 51 Rdnr. 33 f.; Thomas M. Franck, Recourse to Force, Fifth printing, Cambridge 2004, S. 54, 67.

94 Hillgenberg, »Gewaltverbot: Was gilt noch?« aaO. (FN 8), S. 141 (156).

95 Dahm / Delbrück / Wolfrum, Völkerrecht, Bd. I/3, aaO. (FN 12), \$169 IX 9 (S. 825).

96 Dinstein, »Comments on the Presentations by Nico Kirsch and Carsten Stahn« aaO. (FN 8), S. 915 (920). 


\section{Die Zulässigkeit präemptiver oder präventiver Anwendung militärischer Gewalt}

Der Irak-Krieg im Jahre $2003^{97}$ und die sog. Bush-Doktrin ${ }^{98}$ haben die Diskussion über die Zulässigkeit präventiver bzw. präemptiver Selbstverteidigung intensiviert.9 Die USA nehmen das Recht zur Anwendung von militärischer Gewalt bereits bei einer abstrakten Gefahr für sich in Anspruch. ${ }^{100}$ Die sog. Bush-Doktrin ist im völkerrechtlichen Schrifttum auf heftige Kritik gestoßen.101

Die Terminologie in der Auseinandersetzung ist aber nicht einheitlich. ${ }^{102}$ Vielfach wird die völkerrechtlich zulässige präemptive Verteidigung - gemeint ist die Abwehr eines unmittelbar drohenden oder bevorstehenden Angriffs - von der völkerrechtlich unzulässigen präventiven Verteidigung - darunter wird die Abwehr eines

97 Zur Operation »Iraqi Freedom «vgl. Neuhold, »Law and Force in International Relations - European and European and American Positions« aaO. (FN 46), S. 263 (274 ff. m. w. N.); Schaller, »Massenvernichtungswaffen und Präventivkrieg - Möglichkeiten der Rechtfertigung einer militärischen Intervention im Irak aus völkerrechtlicher Sicht « aaO. (FN 31), S. 641 ff.; Christian Tomuschat, »Völkerrechtliche Aspekte des KosovoKonflikts « in: Die Friedenswarte 2003, S. 141 ff.; Michael Bothe, »Der Irak-Krieg und das völkerrechtliche Gewaltverbot « in: Archiv des Völkerrechts 41 (2003), S. 255 ff.

98 »National Security Strategy vom 20. September 2002 «www.whitehouse.gov/nsc/ nss.pdf; dazu Stein / von Buttlar, Völkerrecht, aaO. (FN 1), Rdnr. 824 ff.; Herdegen, Völkerrecht, aaO. (FN 4), $\$ 34$ Rdnr. 4 m. w. N.

99 Vgl. Oskar Matthias Frhr. von Lepel, »Die präemptive Selbstverteidigung im Lichte des Völkerrechts « in: Humanitäres Völkerrecht - Informationsschriften 2003, S. 77 ff.; Streinz, »Wo steht das Gewaltverbot heute? «aaO. (FN 34), S. 219 (221, 226 f.); Holger P. Hestermeyer, »Die völkerrechtliche Beurteilung des Irakkriegs im Lichte transatlantischer Rechtskulturunterschiede« in: Zeitschrift für ausländisches öffentliches Recht und Völkerrecht 64 (2004), S. 315 (326 ff.); Dieter Blumenwitz, »Der Präventivkrieg und das Völkerrecht « in: Politische Studien, Heft 391, 2003, S. 21 ff.; ders., »Die völkerrechtlichen Aspekte des Irak-Konflikts« in: Zeitschrift für Politik 50 (2003), S. 301 (315 ff.); Richard Häußler, »Irak-Krieg und Völkerrecht« in: Nene Zeitschrift für Webrrecht 2004, S. 221 (228 ff.).

100 Vgl. »The National Security Strategy of the United States of America « www.whitehouse. gov/nsc/nss.pdf.; Nico Krisch, »Amerikanische Hegemonie und liberale Revolution im Völkerrecht « in: Der Staat Bd. 43 (2004), S. 267 (272 m. w. N.); Schaller, »Das Friedenssicherungsrecht im Kampf gegen den Terrorismus« aaO. (FN 13), S. 19.

101 Dietrich Murswiek, »Die amerikanische Präventivkriegsstrategie und das Völkerrecht « in: Neue Juristische Wochenschrift 2003, S. 1014 (1019); Theodor Schweisfurth, "Aggression« in: Frankfurter Allgemeine Zeitung vom 28. April 2003; Hillgenberg, »Gewaltverbot: Was gilt noch?« aaO. (FN 8), S. 141 (166 f.); Christian Tomuschat, »Iraq - Demise of International Law? in: Die Friedenswarte 78 (2003), S. 141 (142 f., 158); Thomas Breitwieser, »Vorweggenommene Selbstverteidigung und das Völkerrecht« in: Neue Zeitschrift für Webrrecht 2005, S. 45 ff.; Thomas Bruha / Christian J. Tams, "Die Vereinten Nationen und das Völkerrecht" in: Aus Politik und Zeitgeschichte: Beil. zur Wochenzeitung Das Parlament 55 (2005), 22 vom 30.05.2005, S. 32 (35 ff.).

102 Vgl. Karl-Heinz Kamp, »'Preemptive Strikes'. Eine neue sicherheitspolitische Realität« in: Internationale Politik 6/2004, S. 42 (43). 
nicht unmittelbar bevorstehenden oder drohenden Angriffs oder die Abwehr einer nicht nahen Gefahr verstanden - unterschieden. ${ }^{103}$

Der Tatbestand des Art. 51 SVN ist erst »im Falle eines bewaffneten Angriffs« erfüllt. Regelmäßig löst nur ein konkreter Angriff das Selbstverteidigungsrecht aus. ${ }^{104}$ Nach dem herkömmlichen Verständnis des Wortlauts der Vorschrift heißt dies, dass tatsächlich ein bewaffneter Angriff stattgefunden haben muss. ${ }^{105}$ Von einer Aggression kann dann nicht die Rede sein, wenn sich feindliche Planungen erst in einem Vorbereitungsstadium befinden. Das Selbstverteidigungsrecht setzt danach grundsätzlich die Anwendung von Waffengewalt oder eine gleichwertige militärische Operation voraus. ${ }^{106}$ Jede Art antizipatorischer Selbstverteidigung wäre danach ausgeschlossen. ${ }^{107}$

Freilich muss der zur Selbstverteidigung entschlossene Staat nicht auf den ersten Schuss warten. ${ }^{108}$ Selbstverteidigung ist in extremen Ausnahmelagen dann nicht ausgeschlossen, wenn »der Gegner hin zu einem von ihm geplanten bewaffneten Angriff nur noch ein letztes Schrittchen zurückzulegen hat und der Aufmarsch seiner Truppen bereits vollzogen ist «. ${ }^{109}$ Es ist keinem Staat zuzumuten, die volle Entfaltung eines völkerrechtswidrigen bewaffneten Angriffs hinzunehmen. ${ }^{110}$ Wenn es

103 Vgl. United Nations, General Assembly, 2 December 2004, A more secure world: our shared responsibility, Report of the High-level Panel on Threats, Challenges and Change, A/59/565, Ziff. 189; Eick, »'Präemption', 'Prävention' und die Weiterentwicklung des Völkerrechts « aaO. (FN 50), S. 200 f.; nicht selten wird - statt der Unterscheidung zwischen präemptiver und präventiver Verteidigung - die präventive Verteidigung unter besonderen Umständen für zulässig angesehen, während sie im Regelfall unzulässig sein soll. Stein / von Buttlar, Völkerrecht, aaO. (FN 1), Rdnr. 825, verwenden die Begriffe Präemption und Prävention mit umgekehrter Bedeutung. Wie hier: Kamp, »Preemptive Strikes. Eine neue sicherheitspolitische Realität« aaO. (FN 102), S. 42 (43); zur Staatspraxis: von Buttlar, »Rechtsstreit oder Glaubensstreit? Anmerkungen zur Debatte um die präventive Selbstverteidigung im Völkerrecht«aaO. (FN 29), S. 15 (18ff.)

104 Wolfrum, »Irak - eine Krise auch für das System der kollektiven Sicherheit« aaO. (FN 11), S. 1 (5).

105 Tomuschat, »Völkerrechtliche Aspekte bewaffneter Konflikte« aaO. (FN 58); ders., »Der selbstverliebte Hegemon. Die USA und der Traum von einer unipolaren Welt« in: Internationale Politik 5/2003, S. 39 (40).

106 Dieter Blumenwitz, »Die amerikanische Präventionsstrategie im Lichte des Völkerrechts « in: Horst Fischer / Ulrike Froissart / Wolff Heintschel von Heinegg / Christian Raap (Hg.), Krisensicherung und Humanitärer Schutz - Crisis Management and Humanitarian Protection, Festschrift für Dieter Fleck, Berlin 2004, S. 23 (34).

107 So Randelzhofer, »The Charter of the United Nations « aaO. (FN 10), Art. 51 Rdnr. 34; Antonio Cassese, International Law, 2001, S. 307 ff.; Kugelmann, »Die völkerrechtliche Zulässigkeit von Gewalt gegen Terroristen« aaO. (FN 42), S. 376 (381).

108 Blumenwitz, »Die amerikanische Präventionsstrategie im Lichte des Völkerrechts« aaO. (FN 106), S. 23 (34); ders., »Die völkerrechtlichen Aspekte des Irak-Konflikts« aaO. (FN 53), S. 301 (320); Wiefelspütz, Das Parlamentsheer, aaO. (FN 30), S. 273.

109 Tomuschat, »Präventivkrieg zur Bekämpfung des internationalen Terrorismus? «aaO. (FN 51), S. 121 (127); vgl. auch die kritische Übersicht bei von Buttlar, »Rechtsstreit oder Glaubensstreit? Anmerkungen zur Debatte um die präventive Selbstverteidigung im Völkerrecht« aaO. (FN 29), S. 15 (22 ff.).

110 Doehring, Völkerrecht, aaO. (FN 34), \11 Rdnr. 574; Christian Tomuschat, »International Law: Ensuring the Survival of Mankind on the Eve of a New Century « in: Hague Academy of International Law, 2001, S. 207 (216 f.). 
evident ist, dass ein gewaltsamer Angriff gegen das Staatsgebiet bevorsteht, können Abwehrmaßnahmen beginnen. Selbstverteidigung ist danach bereits dann zulässig, wenn der Gegner den Angriff eingeleitet hat. ${ }^{111}$

Es sind unverkennbar Sachverhalte denkbar, bei denen sich die drohende Angriffsgefahr nicht nur als abstrakte Gefahr manifestiert, sondern sich so sehr verdichtet und konkretisiert, »dass ein Überfall durch den - immer noch potentiellen Gegner nicht mehr als bloße hypothetische Möglichkeit, sondern als ein konkret vorhersehbarer, gleichsam unabwendbarer Geschehensablauf erscheint «. ${ }^{12}$ In solchen Situationen ist es für das Opfer nicht zumutbar abzuwarten, bis die Aggressionsabsicht sich verwirklicht. Vor diesem Hintergrund wird inzwischen überwiegend die präemptive Selbstverteidigung grundsätzlich anerkannt. ${ }^{113}$

Es gibt aber nach wie vor namhafte Autoren, die vor allem wegen der unverkennbaren Missbrauchgefahr Bedenken gegen die vorbeugende Selbstverteidigung geltend machen oder antizipatorisches militärisches Handeln generell ablehnen. ${ }^{14}$ Bereits die Möglichkeit, gegen einen unmittelbar bevorstehenden Angriff militärisch vorzugehen, stelle eine problematische Erweiterung des Selbstverteidigungsrechts dar. Mit einem erfolgten Angriff könne aber nicht die noch nicht konkretisierte Möglichkeit eines Angriffs gleichgesetzt werden; auf keinen Fall rechtfertige sie die Verletzung der territorialen Integrität des Gegners, die mit der vorsorglichen Selbstverteidigung notwendig einher gehe. Der entscheidende Gesichtspunkt gegen jede Form der vorsorglichen Selbstverteidigung liege letztlich in der rein subjektiven Einschätzung des Staates, der Selbstverteidigung geltend mache. Damit sei das Gewaltverbot praktisch zur Disposition dieses Staates gestellt. ${ }^{115}$

Die vor allem im völkerrechtlichen Schrifttum im Zusammenhang mit dem IrakKrieg des Jahres 2003 intensivierte Diskussion über die Zulässigkeit des Präventivkrieges belegt, dass vorsorgliche militärische Maßnahmen nur unter sehr engen Voraussetzungen als zulässig gewertet werden. ${ }^{116}$ Maßstab für die Zulässigkeit präemp-

111 Blumenwitz, »Die amerikanische Präventionsstrategie im Lichte des Völkerrechts« aaO. (FN 106), S. 23 (34); ders., »Der Präventivkrieg und das Völkerrecht« aaO. (FN 99), S. 21 (27).

112 Tomuschat, »Völkerrechtliche Aspekte bewaffneter Konflikte« aaO. (FN 58), S. 450 (460).

113 Oscar Schachter, »The Right of States to Use Armed Force in: Michigan Law Review 82 (1984), S. 1620 (1634 f.); Rosalyn Higgins, Problems and process: international law and how we use it, Oxford 1994, S. 242; Georg Nolte, "Weg in eine neue Rechtsordnung in: Frankfurter Allgemeine Zeitung vom 10. Januar 2003; Wolff Heintschel von Heinegg, "Das maritime ius in bello im 21. Jahrhundert in: Jochen Abr. Frowein / Klaus Scharioth / Ingo Winkelmann / Rüdiger Wolfrum, (Hg.), Verhandeln für den Frieden - Negotiating for Peace, Liber Amicorum Tono Eitel, Berlin u. a. 2003, S. 533 (550 f.).

114 Randelzhofer, »The Charter of the United Nations « aaO. (FN 10), Art. 51 Rdnr. 39 m. w. N.; Horst Fischer, "Zwischen autorisierter Gewaltanwendung und Präventivkrieg: Der völkerrechtliche Kern der Debatte um ein militärisches Eingreifen gegen den Irak « in: Humanitäres Völkerrecht - Informationsschriften 2003, S. 4 (5 f.).

115 Wolfrum, »Irak - eine Krise auch für das System der kollektiven Sicherheit « aaO. (FN 11), S. 1 (6). 
tiver militärischer Maßnahmen ist die vom völkerrechtlichen Schrifttum ganz überwiegend getragene »Webster-Formel $\ll^{117}$ aus dem sog. »Caroline Case $\ll^{118}$. Danach ist antizipatorische Selbstverteidigung nur und frühestens zulässig, wenn die Gefahrenlage »gegenwärtig und überwältigend « ist und »keine Wahl der Mittel und keinen Augenblick zur Überlegung lässt « (»the necessity is instant, overwhelming and leaving no choice of means, and no moment for deliberation « $)^{119}$. Auch die Bundesregierung ist offenbar dieser Auffassung. ${ }^{120}$

Diese Voraussetzungen sind nicht gegeben, wenn nur eine generelle Bedrohungslage, bloße Gefahrenlagen, ${ }^{121}$ ein lediglich möglicher Angriff in eher fernerer $\mathrm{Zu}$ kunft ${ }^{122}$, fern liegende Bedrohungen ${ }^{123}$ oder nur die abstrakte Gefahr eines in unbestimmter Zukunft möglicherweise drohenden Angriffs gegeben ist oder gar nur »Risikovorbeugung « ${ }^{124}$ Zweck der militärischen Unternehmung ist. Dies gilt selbst dann, wenn der Angriff mit Massenvernichtungswaffen erfolgen kann. Ebenso genügen das Gefühl des Bedrohtseins oder frühere Gewalttaten nicht. Die gewaltsame Reaktion auf Friedensbedrohungen unterhalb der Schwelle eines unmittelbar bevorstehenden bewaffneten Angriffs ist ausschließlich Sache des Sicherheitsrats. ${ }^{125}$ Ein pauschaler Verdacht, den internationalen Terrorismus zu unterstützen, erfüllt nicht annähernd die Voraussetzungen für die Wahrnehmung des Rechts auf Selbstverteidigung. ${ }^{126}$ Erst recht ist zur Rechtfertigung von Verteidigungsmaßnahmen nicht ausreichend, wenn ein eher vager Verdacht gehegt wird, dass ein Land eines Tages zu einer konkreten Gefahr für den Weltfrieden werden könnte. ${ }^{127}$ Hartmut

116 Vgl. Geiger, »Grundgesetz und Völkerrecht«aaO. (FN 5), $\mathbb{S} 66$ I (S. 369); Michael E. Kurth, »Der dritte Golf-Krieg aus völkerrechtlicher Sicht« in: Zeitschrift für Rechtspolitik 2003, S. $195 \mathrm{ff}$.

117 Delbrück, »The Fight Against Global Terrorism: Self-Defense or Collective Security as International Police Action? Some Comments on the International Legal Implications of the 'War Against Terrorism' « $\mathrm{aaO}$ (FN 7), 44 (2001), S. 9 (16).

118 Instruktiv: Breitwieser, »Vorweggenommene Selbstverteidigung und das Völkerrecht « aaO. (FN 101), S. 45 (46 ff.); Stein / von Buttlar, Völkerrecht, aaO. (FN 1), Rdnr. 829 f.

119 State Secretary Webster, British and Foreign State Papers 29 (1840 - 1841), S. 1129 (1138).

120 In der Antwort der Bundesregierung (BT-Drs. 15/3181, S. 25) auf eine Kleine Anfrage von Abgeordneten der CDU/CSU heißt es: »Das Recht zur individuellen oder kollektiven Selbstverteidigung nach Art. 51 VN-Charta schließt nach Ansicht der Bundesregierung Abwehrmaßnahmen gegen einen unmittelbar bevorstehenden Angriff ein."

121 Tomuschat, »Präventivkrieg zur Bekämpfung des internationalen Terrorismus?« aaO. (FN 51), S. 121 (127).

122 Neuhold, »Law and Force in International Relations - European and European and American Positions« aaO. (FN 46), S. 263 (273).

123 Fassbender, »Die Gegenwartskrise des völkerrechtlichen Gewaltverbotes vor dem Hintergrund der geschichtlichen Entwicklung « aaO. (FN 3), S. 241 (250); Hillgruber, 》Völkerrechtsprechung «aaO. (FN 17), S. 819.

124 Eick, »'Präemption', ,Prävention' und die Weiterentwicklung des Völkerrechts« aaO. (FN 50), S. 200 (201 f.).

125 Hillgruber, »Völkerrechtsprechung «aaO. (FN 17), S. 819.

126 Dederer, »Krieg gegen Terror« aaO. (FN 13), S. 421 (429).

127 Tomuschat, »Der selbstverliebte Hegemon. Die USA und der Traum von einer unipolaren Welt«aaO. (FN 105), S. 40 (41); Wiefelspütz, Das Parlamentsheer, aaO. (FN 30), S. 276. 
Hillgenberg betont mit Recht: »Die Zulässigkeit von Präventivschlägen ohne ,instant and overwhelming necessity', die , no choice of means and no moment of deliberation’ zulässt, würde das Gewaltverbot als ,Herz' der VN-Charta und Ergebnis aus Erfahrungen mit Weltkriegen aus den Angeln heben. «128

Erfüllen militärische Drohungen nicht die engen Voraussetzungen der WebsterFormel, ist allein der Sicherheitsrat der VN befugt, Maßnahmen zu Friedenssicherung anzuwenden. ${ }^{129}$ Für (zulässige) Verteidigungshandlungen bei einer sich konkret realisierenden Angriffsgefahr hat Yoram Dinstein ${ }^{130}$ den prägnanten Begriff »interceptive strike - Abfangschlag - gefunden, der diesen Sachverhalt überzeugend und anschaulich umschreibt. Diese Betrachtungsweise ist auf Ablehnung ${ }^{131}$ gestoßen, sie findet aber wachsende Zustimmung. ${ }^{132}$

Unverkennbar bereitet es erhebliche Schwierigkeiten, die extreme Gefährlichkeit von Massenvernichtungswaffen im Zusammenhang mit präemptiver Verteidigung angemessen zu würdigen und gleichzeitig die Verletzung des Gewaltverbots durch eine missbräuchliche Inanspruchnahme des Selbstverteidigungsrechts $\mathrm{zu}$ verhindern. ${ }^{133}$ Das Völkerrecht stößt hier angesichts existentieller Interessen von Staaten an seine Grenzen. Bereits der einmalige Einsatz von Massenvernichtungswaffen kann einen unermesslichen Schaden anrichten, ja die Existenz des angegriffenen Staates vernichten. Wenn in einer solchen Situation Verteidigung nicht rechtzeitig einsetzt, ist sie unter Umständen überhaupt nicht mehr möglich. ${ }^{134}$

Eine Grenzziehung zur unzulässigen Gewaltanwendung ist gleichwohl unverzichtbar. Die Diskussion ist ersichtlich noch nicht abgeschlossen. Karl-Heinz Kamp schlägt folgende Kriterien für die Rechtmäßigkeit eigenen militärischen Handelns vor: Die gegnerische Absicht, Schaden zuzufügen muss evident sein. Es müssen Maßnahmen und Vorbereitungen erkennbar sein, dass diese Absicht in die Tat umgesetzt werden soll. Es muss offensichtlich sein, dass ein Nichthandeln das eigene Risiko dramatisch erhöht. ${ }^{135}$

Nach Dieter Dörr und Dorit Bosch könnte in Fällen atomarer Bedrohung ein (zulässiges) präventives Selbstverteidigungsrecht bejaht werden, wenn die Zweit-

128 Hillgenberg, »Gewaltverbot: Was gilt noch?«aaO. (FN 8), S. 141 (166).

129 Blumenwitz, »Der Präventivkrieg und das Völkerrecht« aaO. (FN 99), S. 21 (27).

130 Yoram Dinstein, War, Aggression and Self-Defence, 3. Aufl., Cambridge 2001, S. 172.

131 Vgl. Antonio Cassese, International Law, 2001, S. 309.

132 Derek W. Bowett, Self-Defence in International Law, Manchester 1958, S. 185 f.; Franck, "Recourse to Force« aaO. (FN 93), S. 99 ff; offen gelassen von Tomuschat, »Völkerrechtliche Aspekte bewaffneter Konflikte« aaO. (FN 58), S. 450 (460).

133 Vgl. Frowein, »Ist das Völkerrecht tot? «aaO. (FN 83); Schaller, »Massenvernichtungswaffen und Präventivkrieg - Möglichkeiten der Rechtfertigung einer militärischen Intervention im Irak aus völkerrechtlicher Sicht« aaO. (FN 31), S. 641 (659 f.); Kamp, »Preemptive Strikes. Eine neue sicherheitspolitische Realität« aaO. (FN 102), S. 42 (45).

134 Vgl. Kamp, "Preemptive Strikes. Eine neue sicherheitspolitische Realität« aaO. (FN 102), S. 42 f.; Schaller, »Das Friedenssicherungsrecht im Kampf gegen den Terrorismus « aaO. (FN 13), S. 18.

135 Kamp, »Preemptive Strikes. Eine neue sicherheitspolitische Realität« aaO. (FN 102), S. 46. 
schlag-Kapazität des bedrohten Staates nicht mehr gegeben sei. Es sei unzumutbar, dass eine durch einen unmittelbaren Angriff drohende Zerstörung, die bereits beim ersten Waffeneinsatz des Gegners eintreten könne, abgewartet werden müsse, bevor dagegen militärisch vorgegangen werde. ${ }^{136}$

In Analogie zum Gefahrenabwehrrecht erwägt Oliver Dörr die Wahrscheinlichkeit und den Umfang des potentiellen Schadens beim bedrohten Rechtsgut in Beziehung zum Zeitpunkt von zulässigen Abwehrmaßnahmen zu setzen. ${ }^{137}$ Je gravierender die mutmaßliche Bedrohung, je schwieriger die Angriffsabwehr bei weiterem Zuwarten, je kleiner das »Zeitfenster « für ein effektives Eingreifen, desto weiter müsse ein Staat befugt sein, den drohenden militärischen Angriff zu antizipieren. ${ }^{138}$

Michael N. Schmitt unterbreitete den Vorschlag, Selbstverteidigungsmaßnahmen bereits vor Abschluss der Angriffsvorbereitungen dann zuzulassen, wenn sich anderenfalls das »Fenster effektiver Verteidigungsmöglichkeiten « schlösse. ${ }^{139}$ Andererseits wird der bloße Besitz von Massenvernichtungswaffen zur Rechtfertigung von Verteidigungsmaßnahmen nicht ausreichen. ${ }^{140}$ Das verbietet sich bereits deshalb, weil der Besitz von Massenvernichtungswaffen für sich genommen nicht völkerrechtswidrig ist und im übrigen zu viele Staaten im Besitz solcher Waffen sind.

Militärische Abwehrmaßnahmen sind als Selbstverteidigung dann erlaubt, wenn sich an Bord eines Schiffes Massenvernichtungswaffen, radioaktives Material oder waffenfähige Substanzen befinden, die nachweislich für terroristische Umtriebe bestimmt sind. Solche Einsatzmittel stellen im Besitz von Terroristen immer eine unmittelbare und gegenwärtige Gefahr für den bedrohten Staat dar. ${ }^{141}$

Nach Jochen A. Frowein ist Voraussetzung für die Inanspruchnahme des Selbstverteidigungsrechts, »dass vor der Weltöffentlichkeit im Sicherheitsrat wirklich mit hoher Plausibilität diese Gefährdung dargelegt werden kann. $\ll^{142}$ Es müsse detailliert dargelegt werden können, dass der Angriffsabsichten hegende Staat sich die Voraussetzungen für einen Überraschungsangriff schaffen wolle. ${ }^{143}$ Bei terroristischen Organisationen könne kein Zweifel daran bestehen, dass der Besitz von Massenver-

136 Dörr / Bosch, »Der praktische Fall - Völkerrecht: Aufmarsch vor dem Irak «aaO. (FN 13), S. 477 (480 f.).

137 Dörr, »Das völkerrechtliche Gewaltverbot am Beginn des 21. Jahrhunderts - Was bleibt von Art. 2 (4) UN-Charta? «aaO. (FN 13), S. 33 (45).

138 Dörr, "Das völkerrechtliche Gewaltverbot am Beginn des 21. Jahrhunderts - Was bleibt von Art. 2 (4) UN-Charta? «aaO. (FN 13), S. 33 (45); ähnlich Schaller, »Das Friedenssicherungsrecht im Kampf gegen den Terrorismus« aaO. (FN 13), S. 18.

139 Schmitt, »Preemptive Strategies in International Law « aaO. (FN 50), S. 513 (535).

140 Frowein, »Der Terrorismus als Herausforderung für das Völkerrecht« aaO. (FN 43), S. 879 (889 f.); Schaller, »Das Friedenssicherungsrecht im Kampf gegen den Terrorismus « aaO. (FN 13), S. 19.

141 Christian Schaller, Die Unterbindung des Seetransports von Massenvernichtungswaffen, SWP-Studie, Stiftung Wissenschaft und Politik, Deutsches Institut für Internationale Politik und Sicherheit, Berlin 2004, S. 18, 21.

142 Frowein, »Ist das Völkerrecht tot? «aaO. (FN 83).

143 Stanimir A. Alexandrov, Self-Defense Against the Use of Force in International Law, The Hague u. a. 1996, S. 163 f.; Frowein, »Der Terrorismus als Herausforderung für das Völkerrecht«aaO. (FN 43), S. 879 (891). 
nichtungswaffen als eine unmittelbare Bedrohung anzusehen sei. Es sollte auch nicht bezweifelt werden, dass aus dem Verhalten von Staaten bei Herstellung, Erwerb oder versuchtem Erwerb von Massenvernichtungswaffen der Schluss gezogen werden könne, dass hier eine unmittelbare Bedrohung vorliege. ${ }^{144}$

In diesem Zusammenhang verdient ein aktuelles Dokument der Vereinten Nationen wegen seines prägnanten und repräsentativen Charakters besondere Beachtung. Am 2. Dezember 2004 veröffentlichten die VN den »Report of the High-level Panel on Threats, Challenges and Change - A more secure world: our shared responsibility - A/59/565". ${ }^{145}$ Die »Hochrangige Gruppe« war ein Jahr zuvor von Generalsekretär Kofi Annan eingesetzt worden, um Vorschläge zur Reform des kollektiven Sicherheitssystems und der Vereinten Nationen zu erarbeiten. Zu Art. 51 SVN heißt es dort:

»188. Der Wortlaut dieses Artikels ist restriktiv: Diese Charta beeinträchtigt im Falle eines bewaffneten Angriffs gegen ein Mitglied der Vereinten Nationen keineswegs das naturgegebene Recht zur individuellen oder kollektiven Selbstverteidigung, bis der Sicherheitsrat die zur Wahrung des Weltfriedens und der internationalen Sicherheit erforderlichen Maßnahmen getroffen hat«. Indessen kann ein bedrohter Staat nach lange etablierten Regeln des Völkerrechts militärische Maßnahmen ergreifen, solange der angedrohte Angriff unmittelbar bevorsteht, durch kein anderes Mittel abzuwenden ist und die Maßnahmen verhältnismäßig sind. Ein Problem entsteht dann, wenn die fragliche Gefahr nicht unmittelbar droht, aber dennoch als real dargestellt wird, beispielsweise der in mutmaßlich feindseliger Absicht erfolgende Erwerb der Fähigkeit zur Herstellung von Nuklearwaffen.

189. Kann ein Staat, ohne sich an den Sicherheitsrat zu wenden, unter diesen Umständen das Recht für sich beanspruchen, in antizipatorischer Selbstverteidigung nicht nur präemptiv (gegen eine unmittelbar drohende oder nahe Gefahr) sondern präventiv (gegen eine nicht unmittelbar drohende oder nahe Gefahr) zu handeln? Diejenigen, die dies bejahen, vertreten den Standpunkt, dass manche Gefahren (wie z. B. im Besitz einer Kernwaffe befindliche Terroristen) ein so großes Schadenspotential haben, dass man einfach das Risiko nicht eingehen kann, abzuwarten, bis sie zu einer unmittelbaren Bedrohung werden, und dass durch frühzeitigeres Handeln unter Umständen weniger Schaden angerichtet wird (etwa durch die Vermeidung eines nuklearen Schlagabtauschs oder des radioaktiven Niederschlags aus einer Reaktorzerstörung).

190. Um diese Frage kurz zu beantworten: Wenn gute, durch handfeste Beweise erhärtete Argumente für militärische Präventivmaßnahmen vorliegen, so sollten diese dem Sicherheitsrat unterbreitet werden, der die Maßnahmen sodann nach seinem Gutdünken genehmigen kann. Tut er dies nicht, besteht per definitionem Zeit genug, um andere Strategien zu verfolgen, darunter Überzeugungsarbeit, Verhandlungen, Abschreckung und Eindämmungspolitik, und danach die militärische Option erneut zu prüfen.

144 Frowein, »Ist das Völkerrecht tot?«aaO. (FN 83).

145 Dazu Wiefelspütz, Das Parlamentsheer, aaO. (FN 30), S. 279 f.; Sebastian Graf von Einsiedel, »Vision mit Handlungsanweisung: das High-level Panel und die Reformagenda der Vereinten Nationen« in: Vereinte Nationen 2005, S. 5 ff. 
191. Denjenigen, die einer solchen Antwort mit Ungeduld begegnen, muss entgegengehalten werden, dass in dieser Welt voll mutmaßlicher potentieller Bedrohungen die Gefahr für die globale Ordnung und die Norm der Nichtintervention, auf der diese nach wie vor aufbaut, einfach zu groß ist, als dass einseitige Präventivmaßnahmen, im Unterschied zu kollektiv gebilligten Maßnahmen, als rechtmäßig akzeptiert werden könnten. Einem zu gestatten, so zu handeln, bedeutet, es allen zu gestatten. «146

Abschließend heißt es prägnant:

»192. Wir befürworten keine Neufassung oder Neuauslegung des Artikels 51.«147

Der »Report « ist erkennbar eine Reaktion auf den letzten Irak-Krieg und darf wohl als repräsentativ für die in den VN vertretenen (Mehrheits)Auffassungen zum Selbstverteidigungsrecht nach Art. 51 SVN verstanden werden.

\section{Die humanitäre Intervention}

Unter humanitärer Intervention wird im Völkerrecht die Anwendung militärischer Gewalt zur Verhinderung oder Beendigung schwerster Menschenrechtsverletzungen in einem fremden Staat ${ }^{148}$ oder die Rettung eigener Staatsbürger aus einem anderen Staat ${ }^{149}$ verstanden. Liegt das Einverständnis des betroffenen Staates vor, handelt es sich nicht um eine humanitäre Intervention, sondern um eine einverständliche, völkerrechtlich unbedenkliche Gewaltanwendung. ${ }^{150}$

Humanitäre Interventionen, die durch Resolutionen des Sicherheitsrats der VN legitimiert sind, ${ }^{151}$ sind völkerrechtlich weithin anerkannt. ${ }^{152}$ Umstritten indes sind humanitäre Interventionen, die sich nicht auf ein Mandat der VN stützen können. ${ }^{153}$

146 United Nations, General Assembly, 2 December 2004, A more secure world: our shared responsibility, Report of the High-level Panel on Threats, Challenges and Change, A/ 59/565, Nr. 188 ff.

147 United Nations, General Assembly, 2 December 2004, A more secure world: our shared responsibility, Report of the High-level Panel on Threats, Challenges and Change, A/ 59/565, Nr. 192.

148 Vgl. Dieter Deiseroth, in: Dieter C. Umbach / Thomas Clemens, (Hg.), Grundgesetz, Bd. I, Heidelberg 2002, Art. 24 Rdnr. 309; Fischer, Völkerrecht, aaO. (FN 10), $\ 59$ Rdnr. 26.

149 Herdegen, Völkerrecht, aaO. (FN 4), \ 34 Rdnr. 25; Dörr, »Das völkerrechtliche Gewaltverbot am Beginn des 21. Jahrhunderts - Was bleibt von Art. 2 (4) UN-Charta? aaO. (FN 13), S. 33 (46 f.). Stein / von Buttlar, Völkerrecht, aaO. (FN 1), Rdnr. 805, sehen in der Rettung eigener Staatsangehöriger einen ungeschriebenen Rechtfertigungsgrund (für die Anwendung von Gewalt).

150 Deiseroth, Grundgesetz, aaO. (FN 148), Art. 24 Rdnr. 309.

151 Vgl. die humanitären Interventionen aufgrund einer Genehmigung des Sicherheitsrats der VN in Somalia (1992), Ruanda (1994), Haiti (1994) und im ehemaligen Jugoslawien (1993). SC Res. 794 (1992) vom 3. Dezember 1992, SC Res. 929 (1994) vom 22. Juni 1994, SC Res. 940 (1994) vom 31. Juli 1994, SC Res. 836 vom 10. Juni 1993; zur Praxis der VN: Hobe, »NATO-Intervention im Kosovo: Rückkehr zur Lehre vom gerechten Krieg? «aaO. (FN 6), S. 819 (822 ff.).

152 Vgl. Deiseroth, Grundgesetz, aaO. (FN 148), Art. 24 Rdnr. 311.

153 Dahm / Delbrück / Wolfrum, Völkerrecht, Bd. I/3, aaO. (FN 12), $\$ 169$ X 1 (S. 825 f.). 


\section{a) Humanitäre Intervention bei schwerwiegenden Menschenrechtsverletzungen}

\section{a) Die Diskussion der humanitären Intervention}

Als ungeschriebene Ausnahme vom Gewaltverbot wird immer stärker die humanitäre Intervention bei schwerwiegendsten Menschenrechtsverletzungen erörtert. ${ }^{154}$ Freilich kann nicht davon ausgegangen werden, dass sich bereits Völkergewohnheitsrecht herausgebildet hätte, wonach nicht vom Sicherheitsrat der VN autorisierte militärische humanitäre Interventionen als zulässig betrachtet würden. ${ }^{155}$ Das schließt aber nicht aus, dass vor allem unter Berücksichtigung des Schutzes der Menschenrechte humanitäre Interventionen in Übereinstimmung mit Sinn und Zweck der SVN erfolgen können.

Im Wesentlichen wird die völkerrechtlichen Debatte durch zwei grundlegende Auffassungen geprägt: Die eine Seite versteht das Verbot der unilateralen Gewaltanwendung - mit Ausnahme des Rechts auf individuelle oder kollektive Selbstverteidigung - absolut, während andere annehmen, dass es weitere Fälle gibt, in denen Gewaltanwendung zulässig ist. ${ }^{156}$

Teile des völkerrechtlichen Schrifttums äußern sich kritisch oder gar ablehnend insbesondere wegen der Missbrauchsgefahr - zur Zulässigkeit der humanitären Intervention ohne Mandat der VN. ${ }^{157}$ Diejenigen, die sich für ein Verbot der humanitären Intervention aussprechen, berufen sich vor allem auf den Wortlaut der SVN. Das Gewaltverbot des Art. 2 Ziff. 4 SVN wird von Vertretern dieser Auffassung als absolut und unbeschränkt aufgefasst. ${ }^{158}$ Unter der Charta der Vereinten Nationen

154 Vgl. dazu instruktiv Kay Hailbronner, in: Wolfgang Graf Vitzthum (Hg.), Völkerrecht, 3. Aufl., Berlin 2004, 3. Abschnitt Rdnr. 218 ff. m. w. N.; Herdegen, Völkerrecht, aaO. (FN 4), \$ 34 Rdnr. 4 m. w. N.; Doehring, Völkerrecht, aaO. (FN 34), Rdnr. 1008 ff. m. w. N.; Gunter Warg, Von Verteidigung kollektiver Sicherheit. Der Nato-Vertrag auf Rädern, Frankfurt am Main u. a. 2004, S. $114 \mathrm{ff.}$

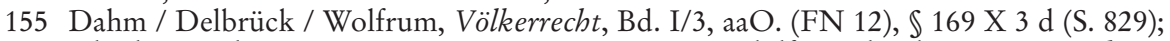
Ulrich Beyerlin, »Humanitarian Intervention« in: Rudolf Bernhardt (Hg.), Encyclopedia Of Public International Law, Vol. 2, Amsterdam 1995, S. 926 ff.; Udo Fink, »Der Kosovo-Konflikt und die Entstehung von Völkergewohnheitsrecht « in: Bernhard Kempen, (Hg.), Die Macht des Geistes: Festschrift für Hartmut Schiedermair, Heidelberg 2001, S. 803 (804); Christian Lange, »Zu Fragen der Rechtmäßigkeit des NATOEinsatzes im Kosovo« in: Europäische Grundrechte-Zeitschrift 1999, S. 313 ff.; Rüdiger Zuck, »Der Krieg gegen Jugoslawien« in: Zeitschrift für Rechtspolitik 1999, S. 225 ff.; Dieter Deiseroth, »'Humanitäre Intervention' und Völkerrecht « in: Neue Juristische Wochenschrift 1999, S. 3084 (3086 f.).

156 Vgl. Wolfrum, »American-European Dialogue: Different Perceptions of International Law - Introduction« aaO. (FN 13), S. 255 (258); einen knappen, aber instruktiven Überblick über die »Denkschulen« bei Stein / von Buttlar, Völkerrecht, aaO. (FN 1), Rdnr. 312 ff.; vgl. auch Kirsten Schmalenbach, »Recht und Gerechtigkeit im Völkerrecht « in: Juristenzeitung 2005, S. 637 ff.

157 Vgl. Josef Isensee, »Weltpolizei für Menschenrechte« in: Juristenzeitung 1995, S. 429 ff.; Randelzhofer, »The Charter of the United Nations" aaO. (FN 10), Art. 2 Ziff. 4 Rdnr. $56-56$ (FN 157 m. w. N.).

158 Vgl. Beyerlin, Encyclopedia Of Public International Law, aaO. (FN 155), S. 926 ff.; Randelzhofer, »The Charter of the United Nations « aaO. (FN 10), Art. 2 Ziff. 4 Rdnr. 57 ff.; Brun-Otto Bryde, »Verpflichtungen Erga Omnes aus Menschenrechten « in: Berichte der Deutschen Gesellschaft für Völkerrecht, Heft 33 (1994), S. 185 f. 
schließe das Gewaltverbot a priori eine humanitäre Intervention mit militärischen Mitteln aus. ${ }^{159}$ Auch wenn zu berücksichtigen sei, dass die Bedeutung des Menschenrechtsschutzes als ein elementarer Bestandteil der völkerrechtlichen Wertordnung ständig zugenommen habe, lasse sich daraus eine Abschwächung der Verpflichtung aus Art. 2 Ziff. 4 SVN aber noch nicht allein begründen. ${ }^{160}$

Sogar die Rettung eigener Staatsangehöriger wird unter Hinweis auf Art. 2 Ziff. 4 SVN für völkerrechtswidrig gehalten. ${ }^{161}$ In der Konsequenz heißt dies, dass selbst im Falle eines Völkermordes gegen den Aggressor nur mit Zustimmung des Sicherheitsrates der VN vorgegangen werden darf und im Falle der Untätigkeit der Vereinten Nationen auch schwersten Menschenrechtsverletzungen hingenommen werden müssen. ${ }^{162}$

Es wird deshalb vor allem von amerikanischen Völkerrechtlern die Auffassung vertreten, die humanitäre Intervention sei unter bestimmten Bedingungen zulässig. ${ }^{163}$ Die Erfahrungen mit den Menschenrechtsverletzungen im ehemaligen Jugoslawien haben aber auch in Europa zu einer Neubewertung der humanitären Intervention geführt, zumal wenn - wie im Fall des Kosovo - der Sicherheitsrat der VN seiner Verantwortung zur Unterbindung schwerster Menschenrechtsverletzungen, begangen von einer Regierung an ihren eigenen Staatsbürgern, nicht nachkommt.

In der Staatengemeinschaft und in der Völkerrechtslehre werden das Gewaltverbot des Art. 2 Ziff. 4 SVN zunehmend - in Deutschland vor allem seit und wegen der deutschen Beteiligung am Kosovo-Krieg im Jahre $1999^{164}$ - nicht als absolute Verbotsnorm verstanden, sondern einer Abwägung mit elementaren Menschenrechten unterzogen. ${ }^{165}$ Oliver Dörr geht nach der humanitären Intervention im Kosovo von einer gewohnheitsrechtlichen Ausnahme vom Gewaltverbot in statu nascendi aus. ${ }^{166}$ Nur ein genuin multilaterales Vorgehen könne in Zukunft gerecht-

159 Wolfrum, »Irak - eine Krise auch für das System der kollektiven Sicherheit «aaO. (FN 11), S. 1 (6); Schmalenbach, »Recht und Gerechtigkeit im Völkerrecht «aaO. (FN 156), S. 637 (642 f.).

160 Dahm / Delbrück / Wolfrum, Völkerrecht, Bd. I/3, aaO. (FN 12), $\$ 169$ X 3 d (S. 829).

161 Randelzhofer, »The Charter of the United Nations « aaO. (FN 10), Art. 2 Ziff. 4 Rdnr. 57.

162 Vgl. Doehring, Völkerrecht, aaO. (FN 34), Rdnr. 1011.

163 Vgl. die Nachweise bei Randelzhofer, »The Charter of the United Nations « aaO. (FN 10), Art. 2 Ziff. 4 FN 146.

164 Vgl. dazu Limpert, »Auslandseinsatz der Bundeswehr« aaO. (FN 34), S. 39 f., 66 ff.; für Michael Wild, »Verfassungsrechtliche Möglichkeiten und Grenzen für Auslandseinsätze der Bundeswehr nach dem Kosovo-Krieg« in: Die öffentliche Verwaltung 2000, S. 622 (628) handelt es sich um eine Militäraktion im Rahmen eines Systems gegenseitiger kollektiver Sicherheit.

165 Knut Ipsen, »Der Kosovo-Einsatz - Illegal? Gerechtfertigt? Entschuldbar? « in: Die Friedens-Warte 1999, S. 19 (20); Karl Doehring, »Die humanitäre Intervention - Überlegungen zu ihrer Rechtfertigung « in: Antônio Augusto Cançado Trindade, (Hg.), The Modern World of Human Rights. Essays in Honour of Thomas Buergenthal, San José 1996, S. 549 (559 ff.); Heiner Willms, »Der Kosovo-Einsatz und das Völkerrecht « in: Zeitschrift für Rechtspolitik 1999, S. 227 ff.; Eckart Klein / Stefanie Schmahl, »Die neue NATO-Strategie und ihre völkerrechtlichen und verfassungsrechtlichen Implikationen « in: Recht und Politik 1999, S. 198 (202 f.)

166 Dörr, »Das völkerrechtliche Gewaltverbot am Beginn des 21. Jahrhunderts - Was bleibt von Art. 2 (4) UN-Charta? «aaO. (FN 13), S. 33 (47); a. A. Fink, 》Der Kosovo-Konflikt und die Entstehung von Völkergewohnheitsrecht«aaO. (FN 155), S. 803 ff. 
fertigt werden. ${ }^{167}$ Claus Kreß erwägt eine Nothilfebefugnis zugunsten einer zu diesem Zweck zum partikularen Völkerrechtssubjekt erhobenen angegriffenen Zivilbevölkerung. ${ }^{168}$ Nach Auffassung von Eckart Klein sind auch einzelne Staaten bei Versagen des Sicherheitsrats zur bewaffneten humanitären Intervention befugt. ${ }^{169}$ Unter engen Voraussetzungen gesteht Jost Delbrück - der Argumentation von Karl Doehring ${ }^{170}$ folgend - ein Recht auf Nothilfe zu. ${ }^{171}$

\section{bb) Bewertung}

Die Diskussion ist erkennbar noch nicht abgeschlossen ${ }^{172}$. Das nicht hinnehmbare ethisch-moralische Desaster, das im Falle der Untätigkeit gegenüber evident schwersten Menschenrechtsverletzungen unausweichlich wäre, gebietet geradezu die Nothilfe. ${ }^{173}$ In diesem Zusammenhang verdient Erwähnung, dass der Sicherheitsrat der VN inzwischen elementare Menschenrechtsverletzungen als Bedrohung des Friedens wertet, da sie geeignet sind, zur Destabilisierung einer Region zu führen. ${ }^{174}$

Die territoriale Integrität eines Regimes, das sich zu »ethnischen Säuberungen« und Völkermord hinreißen lässt, kann nicht der oberste Wert der Völkerrechtsordnung sein. Angesichts schwerster internationaler Verbrechen muss es jedenfalls einer Staatengruppe, die glaubhaft für die Lebensrechte der verfolgten Bevölkerung eintritt, gestattet sein, Gewalt anzuwenden, wenn alle anderen Mittel zur Herstellung eines rechtmäßigen Zustandes versagt haben. ${ }^{175}$

Wegen der erhöhten Missbrauchsgefahr darf die humanitäre Intervention allerdings nur bei gravierendsten Menschenrechtsverletzungen in Betracht gezogen werden. ${ }^{176}$ Nur genozidartige oder vergleichbare systematische Tötungen und Vertreibungen von ganzen Bevölkerungsgruppen können ein Einschreiten mit Waffengewalt rechtfertigen.

167 Dörr, »Das völkerrechtliche Gewaltverbot am Beginn des 21. Jahrhunderts - Was bleibt von Art. 2 (4) UN-Charta?" aaO. (FN 13), S. 33 (48).

168 Kreß, »Jus contra Bellum: Quo vadis?« aaO. (FN 45), S. 62 (65).

169 Klein, »Die Herausforderung durch den internationalen Terrorismus: hört hier das Völkerrecht auf? «aaO. (FN 50), S. 9 (12).

170 Doehring, Völkerrecht, aaO. (FN 34), Rdnr. $1008 \mathrm{ff}$.

171 Delbrück, »Effektivität des UN-Gewaltverbots. Bedarf es einer Modifikation der Reichweite des Art. 2 (4) UN-Charta? «aaO. (FN 59), S. 139 (152 ff.).

172 Vgl. jetzt instruktiv Kreß, »us contra Bellum: Quo vadis? « aaO. (FN 45), S. 62 ff.; Fink, »Der Kosovo-Konflikt und die Entstehung von Völkergewohnheitsrecht« aaO. (FN 155), S. 803 ff.

173 Instruktiv Tomuschat, »Völkerrechtliche Aspekte bewaffneter Konflikte« aaO. (FN 58), S. 450 (455 ff.).

174 Vgl. Resolution Nr. 794 (Somalia) vom 3. Dezember 1992; Resolution Nr. 929 (Ruanda) vom 22. Juni 1994; Resolution Nr. 1199 (Kosovo) vom 23. September 1998.

175 Dupuy / Tomuschat, »Warten auf den Schlag gegen Bagdad « aaO. (FN 9).

176 Tomuschat, »Der 11. September 2001 und seine rechtlichen Konsequenzen« aaO. (FN 22), S. 535 (539). 


\section{b) Humanitäre Intervention zur Rettung eigener oder fremder Staatsangeböriger}

\section{aa) Der Stand der Diskussion}

Ebenfalls völkerrechtlich umstritten ist der unilaterale Einsatz von militärischer Gewalt zur Rettung eigener oder fremder Staatsangehöriger. ${ }^{177}$

Ein nach wie vor erheblicher Teil des Schrifttums hält den unilateralen Einsatz militärischer Gewalt zum Schutz eigener Staatsbürger ohne Zustimmung des betroffenen Staates für nicht völkerrechtskonform. ${ }^{178}$

Gegen die Zulässigkeit eines solchen Vorgehens wird eingewandt, militärische Maßnahmen gegen einen Staat seien immer wieder missbräuchlich damit begründet worden, dass diese der Rettung eigener (oder fremder) Staatsangehöriger dienten. Es treffe nicht zu, dass in der Bedrohung eigener Staatsangehöriger ein bewaffneter Angriff liege, der Selbstverteidigungsmaßnahmen rechtfertige. Es sei nicht möglich, den Angriff auf eine Gruppe von Staatsangehörigen mit einem Angriff auf ein Staatsgebiet gleichzusetzen, denn mit dem Angriff auf die Staatsangehörigen sei keine Gefährdung der territorialen Integrität bzw. der politischen Handlungsfreiheit des Heimatstaates verbunden. ${ }^{179}$ Es überzeuge auch nicht, Befreiungsaktionen auf den Rechtfertigungsgrund der Pflichtenkollision zu stützen. Die Begründung sei eine unzulässige Vorwegnahme des gewünschten Ergebnisses und hebe das Gewaltverbot aus den Angeln..$^{180}$

Es wird demgegenüber weitgehend als realitätsfremd bewertet, einem Staat die gewaltsame Rettung eigener Staatsbürger vor völkerrechtswidriger Bedrohung an Leib und Leben zu versagen. ${ }^{181}$ Schließlich wird die Auffassung vertreten, die Rettung eigener Staatsangehöriger sei u. U. als Selbstverteidigung nach Art. 51 SVN gerechtfertigt. ${ }^{182}$

\section{bb) Bewertung}

Es verdient Zustimmung, dass eine »Völkerrechtslehre, die einem Staat die gewaltsame Rettung eigener Staatsangehöriger vor völkerrechtswidriger Bedrohung an Leib oder Leben versagt, unzumutbare Anforderungen an die Opferbereitschaft des einzelnen Staates ${ }^{183}$ stellt. Erkennbar dringt die Meinung vor, die den gewaltsa-

177 Nach Delbrück, »Effektivität des UN-Gewaltverbots. Bedarf es einer Modifikation der Reichweite des Art. 2 (4) UN-Charta? «aaO. (FN 59), S. 139 (145), zeigt die Einstellung gegenüber der Rechtmäßigkeit des gewaltsamen Schutzes eigener Staatsangehöriger eine gewisse Ambivalenz.

178 Deiseroth, Grundgesetz, aaO. (FN 148), Art. 24 Rdnr. 300 ff. m. w. N.; Dahm / Delbrück / Wolfrum, Völkerrecht, Bd. I/3, aaO. (FN 12), \$169 X 4 (S. 830 ff. m. w. N.).

179 Dahm / Delbrück / Wolfrum, Völkerrecht, Bd. I/3, aaO. (FN 12), $\mathbb{1 6 9}$ X 4 (S. 831 m. w. N.).

180 Weinzierl, »Vorlesungs-Abschlussklausur - Völkerrecht: Geiselbefreiung im Ausland « aaO. (FN 13), S. 602 (605).

181 Herdegen, Völkerrecht, aaO. (FN 4), $\mathbb{3} 34$ Rdnr. 22 m. w. N.; Doehring, »Die humanitäre Intervention - Überlegungen zu ihrer Rechtfertigung «aaO. (FN 165), S. 599 ff..

182 Stahn, »'Nicaragua is dead, long live Nicaragua' - the Right to Self-defence Under Art. $51 \mathrm{UN}-\mathrm{Charter}$ and International Terrorism« aaO. (FN 36), S. 827 (856 f.).

183 Herdegen, Völkerrecht, aaO. (FN 4), \$34 Rdnr. 22. 
men Schutz eigener Staatsangehöriger unter bestimm-ten Voraussetzungen zulässt. ${ }^{184}$ Oliver Dörr meint sogar, es sei eine völkergewohnheitsrechtliche Ausnahme zum Gewaltverbot entstanden. ${ }^{185}$ Allerdings darf dies nicht als Vorwand für politisch motivierte Gewaltaktionen dienen. ${ }^{186}$

\section{Resümee und Ausblick}

Das umfassende Verbot der Anwendung und Androhung militärischer Gewalt und die völkerrechtlich zulässige militärische Reaktion auf eine Verletzung des Gewaltverbots wurden durch schwere Menschenrechtsverletzungen, durch die terroristischen Anschläge vom 11. September 2001, durch den Irak-Krieg und durch die sog. Bush-Doktrin mit neuen Realitäten konfrontiert. Vor diesem weltpolitischen Hintergrund lassen sich unterschiedliche Tendenzen feststellen: Eine vorsichtige Weiterentwicklung des Völkerrechts im Umgang mit terroristischen Anschlägen nichtstaatlicher Organisationen, gleichzeitig aber auch das strikte Festhalten an den etablierten Regeln des Rechts zur individuellen oder kollektiven Selbstverteidigung unter ausdrücklicher Ablehnung der Befugnis zur präventiven Anwendung militärischer Gewalt.

Danach können terroristische Anschläge erheblichen Ausmaßes das Selbstverteidigungsrecht eines Staates auch dann begründen, wenn sie von nichtstaatlichen Organisationen begangen wurden. Hier ist das Völkerrecht angesichts veränderter Bedrohungen durch Staatenpraxis und Völkerrechtswissenschaft weiterentwickelt worden.

Ein bedrohter Staat darf militärische Maßnahmen zur Verteidigung ergreifen, solange der angedrohte Angriff unmittelbar bevorsteht, durch kein anderes Mittel abzuwenden ist und die Maßnahmen verhältnismäßig sind. In der Staatengemeinschaft, aber auch in der Völkerrechtswissenschaft ist mehrheitlich ein striktes Beharren auf Art. 51 SVN in seiner überkommenen Interpretation zu erkennen. Die Grenze zwischen zulässiger präemptiver Gewalt und völkerrechtswidriger präventiver Gewalt wird prinzipiell durch die überkommene Caroline-Formel gezogen.

Umstritten bleibt freilich weiterhin, wie mit nuklearen Gefahrenlagen umzugehen ist. Ebenso wenig ist die Diskussion über die Zulässigkeit der unilateralen humanitären Intervention abgeschlossen.

184 Vgl. Herdegen, Völkerrecht, aaO. (FN 4), \34 Rdnr. 22.

185 Dörr, »Das völkerrechtliche Gewaltverbot am Beginn des 21. Jahrhunderts - Was bleibt von Art. 2 (4) UN-Charta? " aaO. (FN 13), S. 33 (46).

186 Vgl. Herdegen, Völkerrecht, aaO. (FN 4), $\mathbb{S} 34$ Rdnr. 22. 


\section{Zusammenfassung}

Ausnahmen vom Gewaltverbot sind die Zwangsmaßnahmen des Sicherheitsrats der VN und das Recht auf individuelle und kollektive Selbstverteidigung (Art. 51 SVN). Es wird darüber hinaus zunehmend die humanitäre Intervention als Ausnahme vom Gewaltverbot diskutiert.

Das Selbstverteidigungsrecht nach Art. 51 SVN setzt einen »bewaffneten Angriff « auf einen Staat voraus. Insbesondere die terroristischen Anschläge vom 11. September 2001 in den USA haben zu einer Neubewertung der Voraussetzungen für einen bewaffneten Angriff i. S. des Art. 51 SVN geführt. Mittlerweile scheint sich die Auffassung durchgesetzt zu haben, dass auch derjenige Staat als legitimes Ziel der Selbstverteidigung gilt, der private Gewalttäter im weiteren Sinne unterstützt.

Der Irak-Krieg im Jahre 2003 und die sog. Bush-Doktrin haben die Diskussion über die Zulässigkeit präventiver bzw. präemptiver Selbstverteidigung intensiviert. Inzwischen wird überwiegend die präemptive Selbstverteidigung grundsätzlich anerkannt. Danach ist antizipatorische Selbstverteidigung nur und frühestens zulässig, wenn die Gefahrenlage »gegenwärtig und überwältigend « ist und »keine Wahl der Mittel und keinen Augenblick zur Überlegung lässt « (»the necessity is instant, overwhelming and leaving no choice of means, and no moment for deliberation «). Erfüllen militärische Drohungen nicht diese engen Voraussetzungen, ist allein der Sicherheitsrat der VN befugt, Maßnahmen zu Friedenssicherung anzuwenden. Unverkennbar bereitet es aber erhebliche Schwierigkeiten, die extreme Gefährlichkeit von Massenvernichtungswaffen im Zusammenhang mit präemptiver Verteidigung angemessen zu würdigen und gleichzeitig die Verletzung des Gewaltverbots durch eine missbräuchliche Inanspruchnahme des Selbstverteidigungsrechts zu verhindern. Als ungeschriebene Ausnahme vom Gewaltverbot wird immer stärker die humanitäre Intervention bei schwerwiegendsten Menschenrechtsverletzungen erörtert und für zulässig gehalten.

Vor dem aktuellen weltpolitischen Hintergrund lassen sich unterschiedliche Tendenzen feststellen: Eine vorsichtige Weiterentwicklung des Völkerrechts im Umgang mit terroristischen Anschlägen nichtstaatlicher Organisationen, gleichzeitig aber auch das strikte Festhalten an den etablierten Regeln des Rechts zur individuellen oder kollektiven Selbstverteidigung unter ausdrücklicher Ablehnung der Befugnis zur präventiven Anwendung militärischer Gewalt.

\section{Summary}

The exceptions to the ban on the use of force are enforcement measures taken by the UN Security Council and the right of individual and collective self-defence (Article 51 of the UN Charter). In addition, humanitarian intervention is increasingly being discussed as an exception to the ban on the use of force.

The right of self-defence under Article 51 of the UN Charter presupposes an 'armed attack' on a state. The terrorist attacks of 11 September 2001 in the USA, in 
particular, have caused the criteria defining an armed attack for the purposes of Article 51 of the Charter to be reassessed. The view that the state which supports, in the broader sense, the private individuals carrying out the attacks also represents a legitimate target for self-defence now seems to have become accepted.

The war in Iraq in 2003 and the 'Bush doctrine' have intensified the debate on the admissibility of preventive and pre-emptive self-defence. Pre-emptive self-defence is now recognised in principle by most. This states that anticipatory self-defence is permissible only, and at the earliest, when the necessity is »instant, overwhelming and leaving no choice of means, and no moment for deliberation «.

If military threats do not meet these narrow requirements, only the UN Security Council is empowered to take measures to enforce the peace. Unquestionably, however, it is very difficult to take adequate account of the extreme danger posed by weapons of mass destruction in the context of pre-emptive defence and, simultaneously, to prevent the ban on the use of force from being infringed by states misusing the right of self-defence.

Humanitarian intervention in the worst cases of human rights violations is increasingly discussed and considered admissible as an unwritten exception to the ban on the use of force.

Against the background of current political developments at global level, various trends can be identified: international law is cautiously being further developed as regards dealing with terrorist attacks by non-state organisations, but at the same time there is a determination to retain the established rules of the right of individual or collective self-defence, with the power to make preventive use of military force being explicitly rejected. 